\title{
THE TOLMAN SURFACE BRIGHTNESS TEST FOR THE REALITY OF THE EXPANSION. I. CALIBRATION OF THE NECESSARY LOCAL PARAMETERS
}

\author{
Allan Sandage \\ Observatories of the Carnegie Institution of Washington, 813 Santa Barbara Street, Pasadena, CA 91101 \\ AND \\ LORI M. LUBIN ${ }^{1,2}$ \\ Department of Astronomy, Mail Stop 105-24, California Institute of Technology, Pasadena, CA 91125 \\ Received 2000 November 6; accepted 2001 January 30
}

\begin{abstract}
The extensive CCD photometry by Postman \& Lauer in the Cape/Cousins $R$ photometric band for first-ranked cluster elliptical and S0 galaxies in 118 low redshift $(\langle z\rangle=0.037)$ clusters is analyzed for the correlations between average surface brightness, linear radius, and absolute magnitude. The purpose is to calibrate the correlations between these three parameters in the limit of zero redshift. The PostmanLauer cluster galaxies at low redshift approximate this limit. We apply small corrections for the finite mean redshift of the sample in order to define the zero-redshift correlations. These local correlations provide the comparisons to be made in Paper IV with the sample of early-type galaxies at high redshift in search of the Tolman surface brightness signal of $(1+z)^{4}$ if the expansion is real.

Surface brightness averages are calculated at various metric radii in each galaxy in the sample. The definition of such radii by Petrosian uses ratios of observed surface photometric data. Petrosian radii have important properties for the Tolman test which are reviewed in this paper. The observed surface brightnesses are listed for 118 first-ranked cluster galaxies at Petrosian $\eta$ radii of 1.0, 1.3, 1.5, 1.7, 2.0, and $2.5 \mathrm{mag}$. The three local diagnostic correlation diagrams are defined and discussed. We review the Tolman test and show that, although recipes from the standard cosmological model that already have the Tolman signal incorporated are required to calculate linear radii and absolute magnitudes from the observed data, the test is nevertheless free from the hermeneutical circularity dilemma occasionally claimed in the literature. The reasons are the observed mean surface brightness (1) is independent of any assumptions of cosmological model, (2) does not depend on the existence of a Tolman signal because it is calculated directly from the data using only angular radii and apparent magnitudes, and (3) can be used to search for the Tolman signal because it carries the bulk of that signal.
\end{abstract}

Key words: cosmology: observations - galaxies: clusters: general

\section{INTRODUCTION}

Tolman $(1930,1934)$ derived the remarkable result that, in an expanding universe with any arbitrary geometry, the surface brightness of a set of "standard" (identical) objects will decrease by $(1+z)^{4}$. One factor of $(1+z)$ comes from the decrease in the energy of each photon due to the redshift. The second factor comes from the decrease in the number flux per unit time. Two additional factors of $(1+z)$ come from the apparent increase of area due to aberration. The effect is the same for all intrinsic geometries because the cosmological geometric effects due to different space curvatures (i.e., the dependence on $q_{0}$ ) are identical in the equations for luminosity, $L=f\left(q_{0}, z\right)$ and intrinsic radius = $g\left(q_{0}, z\right)$. Hence, the ratio of $L$ to (radius) ${ }^{2}$, which is the surface brightness, is independent of all the cosmological parameters, precisely (Sandage 1961, 1972).

It was realized early that the Tolman prediction constituted a test of the reality of the expansion because the effect would be different in an nonexpanding universe. There, the redshift is presumed to be due either to some unknown

\footnotetext{
${ }^{1}$ Hubble Fellow.

2 Current address: Department of Physics and Astronomy, Johns Hopkins University, Baltimore, MD 21218.
}

property of matter or to interactions with matter in the path length. The surface brightness would then decrease by only one factor of $(1+z)$. Only the energy effect of individual redshifted photons would be present. The aberration and the decrease in the flux of energy flow due to the "number effect" would be zero (Geller \& Peebles 1972; Sandage 1974; Sandage \& Perelmuter 1990a).

Although the test has been known in principle for 70 years, attempts to implement it have been few because four difficult practical problems must first be solved.

1. How does one use an operationally robust definition of average surface brightness for any galaxy class, say, elliptical and S0 galaxies, when the surface brightness of such galaxies is a strong function of position in the image, varying by a factor of 1000 between center and "edge"?

2. How can one define an appropriate radius over which to measure a mean surface brightness in objects with such strong intensity gradients? Further, how does one define a metric size rather than an isophotal size that is independent of the cosmological geometry using only the observed photometric data for the test galaxies? Use of isophotal radii causes a degeneracy for the test (Sandage 1961, 1972, 1995).

3. How does one avoid systematic effects at large redshifts due to the finite resolution of the point-spread functions for the telescopes used to obtain the data? Angular 
resolutions of the order of 0.1 for redshifts larger than $z=0.5$ are required.

4. How does one account for any evolutionary change in surface brightness and/or metric size between the high redshift galaxies and the local galaxies that are used for the comparison due to the appreciable difference in the lookback times?

Problems 1-3 were addressed in three earlier papers (Sandage \& Perelmuter 1990a, 1990b, 1991; hereafter SPI, SPII, and SPIII) where a Tolman signal was claimed, based on a particular set of ground-based data (Djorgovski \& Spinrad 1981, hereafter DS). However, the results of this test were marginal because the angular resolution of the ground-based data was only on the order of $1^{\prime \prime} .0$ and because only photographic data were used. One of the proposed cosmological projects for a large space telescope then in the early planning stage (called the LST at the time) was the Tolman test (Sandage 1974) because the diffraction limit of the LST would be between 0.05 and 0 ". 10 , at least 10 times better than from the ground. After 25 years, we now make a first attempt to carry out the test using data from the Hubble Space Telescope (HST) for three galaxy clusters at high redshift studied in a series of papers using the Keck and the Kitt Peak telescopes and the HST (Oke, Postman, \& Lubin 1998; Postman, Lubin, \& Oke 1998, 2001; Lubin et al. 1998, 2001). These authors have studied nine remote clusters, obtaining spectroscopic redshifts for 892 very faint galaxies with the Keck $10 \mathrm{~m}$ telescopes and $B V R I K$ colors of many galaxies in the fields with both the Keck and the HST.

We have used the Keck and HST spectroscopic and photometric data for three of the clusters $(\mathrm{Cl} 1324+3011$, Cl 1604+4304, and $\mathrm{Cl} 1604+4321$ ). The redshifts of the clusters are $0.76,0.90$, and 0.92 , respectively. We have determined intensity profiles with radii from the HST WFPC2 images where there is sufficient angular resolution to permit reliable Petrosian (1976) angular radii to be measured at Petrosian $\eta$-values from 1.0 to $2.0 \mathrm{mag}$. From these data we have calculated observed surface brightnesses at each of the Petrosian radii, which, when corrected to rest wavelengths by calculated $K$ terms, permit comparison of surface brightnesses of the high-redshift cluster galaxies with similar data for local elliptical (E) and S0 galaxies of known absolute magnitudes.

The present paper is the first of four on this Tolman test using HST data. It is concerned with the calibration of three diagnostic diagrams that define the relations between surface brightness, absolute magnitude, and radii for local early-type galaxies. There are systematic variations between each of these quantities for the manifold of early-type galaxies. These variations must be calibrated out to recover a pure Tolman signal from the observations of the surface brightness of similar high-redshift galaxies as modified by evolution in the look-back time.

The problem is the same as was addressed in SPII where the calibrations of the necessary scaling relations were made using galaxies in the Virgo, Fornax, and Coma clusters. The calibrations were extended in SPIII using additional photographic photometry data on first, second, and thirdranked galaxies in 56 Abell clusters and well-known groups. The data used there were from the surface photometry principally by Oemler (1976), Thuan \& Romanishin (1981),
Malumuth \& Kirshner (1985), and Schombert (1986, 1987).

We have repeated these scaling calibrations in the present paper using the CCD photoelectric photometry of Postman \& Lauer (1995, hereafter PL) for the first-ranked galaxies in 118 Abell clusters. The reasons for repeating the local calibrations are (1) to test the systematic accuracy of the data in SPI, SPII, and SPIII using the increased systematic CCD photoelectric accuracy of the PL data, and (2) to use the PL data in the $R$ band rather than $B$ and $V$ as in the SP series. The observations of the high-redshift galaxies with HST were made in the band passes of F702W and F814W which are closer to the $R$ and $I$ bands than the $V$ band used in SPII and SPIII.

In this first paper of the present series, we set out properties of the Petrosian $\eta$ metric radii and show again why they are so important for the test. The calibration of the three correlations of mean surface brightness, linear radii, and absolute magnitude using Petrosian metric sizes are the main results of the present paper. In Lubin \& Sandage (2001a; hereafter Paper II) we show the sensitivity of the accuracies of Petrosian radii to the size of the point-spread functions for both the Keck ground-based and the HST data. We also address the proper way to reduce profile and surface-brightness data for highly flattened $\mathrm{E}$ galaxies using either circular apertures with $(a b)^{1 / 2}$ effective radii, where $a$ and $b$ are the semimajor and semiminor axes of the best fitting ellipse, or elliptical apertures with the observed ellipticity.

In Lubin \& Sandage (2001b; hereafter Paper III) we determine the profile and surface brightness data for the early-type galaxies in the three high-redshift clusters used here for the test. Lubin \& Sandage (2001c; hereafter Paper IV) use the data from Paper III to make the Tolman test. We discuss there (1) the effects of luminosity evolution in the look-back time and (2) what may appear to be a partial degeneracy of the test where we need knowledge of absolute magnitudes and linear radii that can only come from the Mattig (1958) equations at high redshift. These equations already are based on the standard cosmological model that have the Tolman surface brightness signal built into them. We show in Paper IV that the test is, however, robust and that the observed data are consistent with the Tolman prediction whereas the nonexpanding (tired light) models for nonexpansion are not. In $\S 5$ of the present paper we argue that the test in Paper IV is not circular because the bulk of the Tolman signal is contained in the observed surface brightnesses with only a slight dependence on the linear radii that must be calculated from the assumed cosmology.

\section{PETROSIAN $\eta$ METRIC RADII}

\subsection{Definition of $\eta$ and Properties of the Petrosian $\eta$ Ratio}

Definitions and properties of Petrosian metric radii are derived elsewhere (DS; SPI; Sandage 1995) based either on the intensity profile or the observed growth curve of magnitude versus aperture. We give here only a summary of the definitions and some of the remarkable properties of the function. Defined as twice the slope of the growth curve expressed in magnitudes as

$$
\eta(\mathrm{mag})=2.5 \log \{2 d(\log r) / d[\log L(r)]\},
$$

it is proved in DS, SPI, and originally by Petrosian (1976) that this definition is identical to finding the difference in magnitude between the mean surface brightness, $\langle\mathrm{SB}(r)\rangle$, 
averaged over the area interior to a particular radius and the surface brightness, $\mathrm{SB}(r)$, (i.e., the profile intensity) at that radius. Hence, to find the radius where the surface brightness averaged over the area encompassed by a particular radius is, say, two magnitudes brighter than the surface brightness at that radius, we need only calculate the mean surface brightness for various radii and interpolate to the radius where $\langle\mathrm{SB}\rangle$ is two magnitudes brighter than $\mathrm{SB}(r)$.

The calculations can either be made from a known growth curve using equation (1) if we have aperture photometry with increasing aperture sizes or by integrating a known intensity profile to calculate the mean surface brightness at a series of radii. The integral method using observed profiles was used in SPI. The profile data were obtained from the literature cited in the last section. The equations in SPI for $\eta$ are their equation (6) for the definition, equation (7) for the $\langle\mathrm{SB}\rangle$ averaged over $r(\eta)$, equation (10) for the profile giving the $\mathrm{SB}(r)$ at $r(\eta)$, and equation (13) for $\eta$ expressed in magnitudes.

Because $\eta$ is the ratio of various surface brightnesses in a given galaxy, it defines a metric (rather than an isophotal) radius that is independent of reddening, absorption, redshift, color (if there is no color gradient across the image), luminosity evolution (again if there is no differential evolution across the image), and the $K$-correction term for the effect of redshift.

\subsection{The Two Ways of Calculating $\eta$}

The method of calculating $\eta$ using only the profile is shown in Figure 1, taken from Figure 5 of SPI. The profile data for NGC 3379 are from de Vaucouleurs \& Capaccioli (1979). The intensity contained within each tabulated radius is obtained by numerical integration using "circular aper-

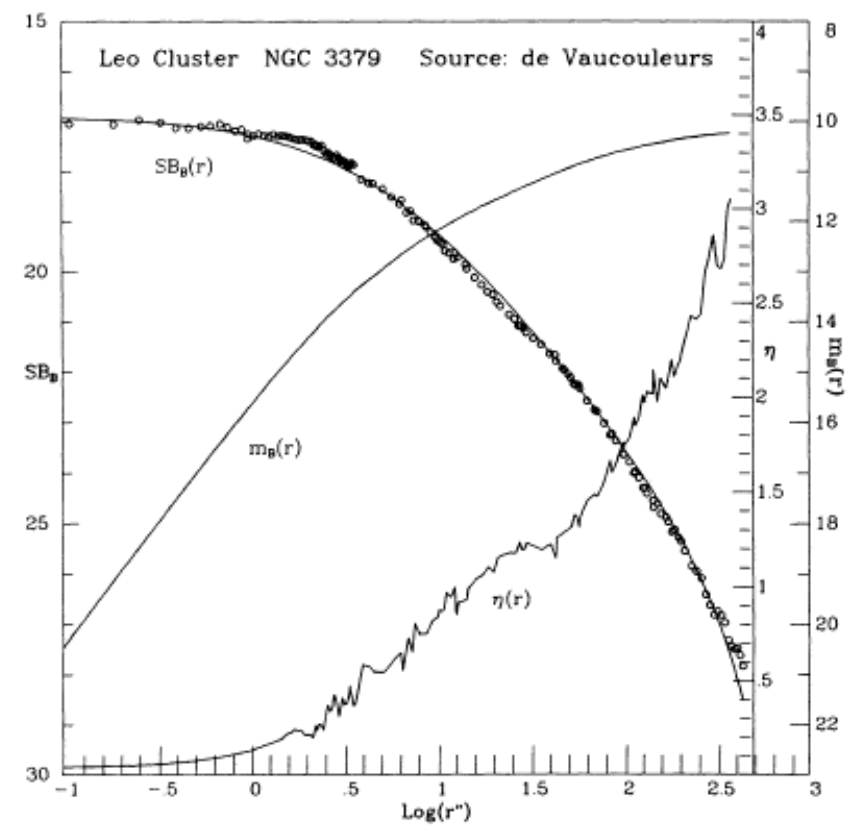

Fig. 1.- Showing how the growth curve, $m(r)$, and the Petrosian $\eta$ parameter, $\eta(r)$, are related to the profile $\mathrm{SB}(r)$. The profile data for NGC 3379 are from de Vaucouleurs \& Capaccioli (1979). The solid line is an Oemler (1976) modified Hubble profile with $\alpha / \beta=70$. The $\eta$-values have been calculated by the integral method in the text for every listed profile point. This diagram is taken from Sandage \& Perelmuter (1990a). tures." At each radius the difference in magnitude between this running integration and the profile value, $\mathrm{SB}(r)$, is calculated. This function is shown in Figure 1 and labeled as $\eta(r)$. Note the ragged nature of the $\eta$ curve, calculated there at every listed radius for which the $\mathrm{SB}(r)$ profile was given by de Vaucouleurs \& Capaccioli (1979). The growth curve, also calculated by integrating the profile within circular apertures, is labeled $m(r)$ in Figure 1.

The calculation of $\eta(r)$ from the slope of the growth curve is often easier than from the profile because the necessary data are contained directly from the aperture photometry. Such data were routinely obtained as the output of the single channel photometers of the 1950s to 1980 s by using various blocking apertures and measuring the magnitude contained within a particular aperture. The various photometric programs for the Hubble diagram in the 1970s on cluster galaxies (e.g., Sandage 1972, 1975) were done that way. No knowledge is needed of the profile, which is, of course, the first derivative of the growth curve.

Growth curve data are also given by PL in their "aperture" photometry of first-ranked galaxies in the 119 nearest Abell clusters. Although they used a CCD areal detector, they list the magnitude inside various circular apertures made by summing the intensities, pixel by pixel, to given radial distances from the image center. Because of the straightforward calculation of the slope of the growth curve directly from the data listed by PL, we have used equation (1) to calculate $\eta(r)$-values from these data.

Adopting the slope of that curve to be the $\alpha$ parameter of $\mathrm{PL}$, which is calculated from the growth curve as

$$
\alpha=0.4 d[m(r)] / d(\log r),
$$

$\eta$ follows from

$$
\eta(\mathrm{mag})=2.5 \log (\alpha / 2)
$$

using equation (1).

\section{THE $\eta$ PARAMETERS FROM THE POSTMAN-LAUER DATA FOR LOCAL FIRST-RANKED ELLIPTICAL AND S0 CLUSTER GALAXIES}

\subsection{Color and Magnitude Systems}

The PL CCD photometric data in the $B$ and $R$ passbands are given in their Table 3 . The photometry in $B$ is on the standard Johnson/Morgan $U B V$ system. The photometry in $R$ is on the Cape-Cousins $R_{\mathrm{C}}$ system which differs by an appreciable color equation from the Johnson (1965; Johnson et al. 1966; also Mendoza 1967) $R_{J}$ system (Sandage 1997, eqs. [1] and [2]). That system, $R_{J}$, is the same as the original $r(\mathrm{~S} 20)$ system of Sandage \& Smith (1963). $R_{J}$ is also the system used for all the Palomar photometry by one of us for the bright cluster galaxies, based on the defining list of standards for that photometry (Sandage $1972,1973 \mathrm{~b}$ ). The nonlinear relation between $R_{J}$ and $R_{\mathrm{C}}$ is given elsewhere (Sandage 1997).

In all the remaining parts of the present series we use the $R_{\mathrm{C}}$ (Cape/Landolt) system as given in the PL photometry based on Landolt $(1983,1992)$ standards for the Cape $(R I)_{\text {Cape }}$ system. However, it must be noted in the comparisons of the PL $R_{\mathrm{C}}$ photometry with the $R_{\mathrm{S} 20}$ photometry of Sandage (1973b) that the difference in the two $R$ systems at the color of elliptical galaxies has a zero point offset of 0.26 mag. The $R_{\mathrm{C}}$ is fainter than $R_{J}$, and, therefore, the $R$ magni- 
tudes in PL are fainter by the same amount at all their aperture sizes from the $R$ magnitudes listed by Sandage (1973b) for the galaxies in common.

\subsection{The Linear Radius, Absolute Magnitude, and Surface Brightness at Various $\eta$ Radii for Local First-ranked Cluster Galaxies}

PL list their equivalent "circular" aperture photometry for each of their program galaxies using equal intervals of $\left(\log r^{\prime \prime}\right)$. They have set their second listed entry for each galaxy at their standard metric radius of $16 \mathrm{kpc}$ (defined with their adopted Hubble constant). With equal intervals of $\log r^{\prime \prime}$, the slope of the growth curve, $\alpha$, is calculated from equation (2) from their radii and magnitude differences directly. $\eta(r)$ at each of the listed radii then follows from equation (3).

We calculated the slope of the growth curve at each listing in the master Postman-Lauer table. This gives the value of $\eta$ at each listed radius. Interpolation gives the radii for $\eta$-values of 1.0, 1.3, 1.5, 1.7, 2.0, and $2.5 \mathrm{mag}$. These are the same intervals of $\eta$ used in SPI, SPII, and SPIII. The magnitude inside each of the interpolated $\eta$-values was then read from the plotted growth curves for each of the $118 \mathrm{PL}$ clusters that have adequate data. The surface brightness, in magnitudes per square arcsecond, is then calculated from

$$
\langle\mathrm{SB}\rangle_{\eta}=2.5 \log \left(\pi r_{\eta}^{2}\right)+m_{\eta},
$$

where $m_{\eta}$ is the apparent magnitude of the light encompassed within the respective $\eta$ radii, $r_{\eta}$ (in arcseconds). Note that this is independent of all cosmology as to "proper distance." It is a directly observed quantity, no matter what its interpretation (see $\S 3.4$ ).

\subsection{Correction of the Observed Postman-Lauer Data for Galactic Absorption and the K Term for the Effect of Redshift}

The observed apparent magnitudes have been corrected for Galactic absorption and the $K(z)$ effect of redshift. We first compared the corrections adopted by PL with previous determinations of the Galactic absorptions, $A(R)$, and the $K(z)$ terms in $R$. The comparison of $K_{z}(R)$ was with the $K(z)$ values used in the earlier photometry of clusters (Sandage 1973a, Table 5). The agreement is excellent for both $K_{z}(R)$ and $K_{z}(B-R)$ at the level of $0.01 \mathrm{mag}$.

The $B-R$ colors listed by Postman-Lauer, corrected by their adopted $K(B-R)$ values, were then correlated with the cosecant of the Galactic latitude and with the $E(B-V)$ reddenings adopted by Lauer \& Postman (1994) from the tables of Burstein \& Heiles (1984). These reddenings were also correlated with the cosecant of the Galactic latitude using the listings of the Galactic coordinates of the clusters in Table 1 of Lauer \& Postman (1994). These correlations are highly consistent with the reddening and absorption model of zero absorption in the pole down to Galactic latitude $45^{\circ}$, and then an increase in $A_{R}$ by 0.08 mag per unit change in cosecant $b$ for csc $>1.5$ adopted in Paper VIII of the velocity-distance series (Sandage 1975). The slope coefficient adopted by PL is $0.09 \mathrm{mag} / \mathrm{csc} b$. In view of the excellent agreement of these data we have adopted the $K(z)$ and $A_{R}$ corrections of PL. The adopted corrections for absorption are $A_{R}=2.35 E(B-V)$, where we have used the reddenings of Lauer \& Postman (1994).

\subsection{Absolute Magnitudes, Linear Radii, and Observed Surface Brightnesses Corrected for Galactic Absorption and $\mathrm{K}(\mathrm{z})$}

The size of the database is very large if we were to include the complete data on the apparent magnitude and angular radius for each $\eta$-value for each cluster. We do not list these observed data here. However, they can be recovered from Table 1 using the listed absolute magnitudes and linear radii, together with the listed $m-M$ moduli and the factor, $f$, that changes angular radii into linear radii, as described below. The data in Table 1 were calculated from the observed apparent magnitudes (corrected for Galactic absorption and $K$ term) and angular radii (in arcseconds) in the following way.

Each Abell cluster is identified in the extreme left-hand column of Table 1. Under the identification Abell cluster number in that column is the adopted cluster redshift from Table 1 of Lauer \& Postman (1994). The adopted distance modulus $m-M$ is given next in that column, based on the redshift and an arbitrary Hubble constant of $50 \mathrm{~km} \mathrm{~s}^{-1}$ $\mathrm{Mpc}^{-1}$. If we use the naive formulation of the velocity distance relation (with no regard for the geometry of different $q_{0}$-values) where the present distance is simply $c z / H_{0}$, then for $H_{0}=50$

$$
m-M=5 \log z+43.891 .
$$

If, on the other hand, we adopt the exact Mattig equation taking into account the intrinsic geometry (i.e., the $q_{0^{-}}$ value), then equation (5) is in error by the magnitude correction of

$$
\Delta(m-M)=1.086\left(1-q_{0}\right) z
$$

(Mattig 1958; Sandage 1961, 1995). This correction will be zero for $q_{0}=1$ and approximately $0.5 z$ mag for $q_{0}=1 / 2$. The redshifts of the Postman-Lauer clusters are all smaller than $z=0.05$, averaging $\langle z\rangle=0.037$; therefore, this implies an average correction of $\sim 0.02 \mathrm{mag}$ to $M$ for $q_{0}=1 / 2$ if equation (5) had been used. To be consistent with our calculation of the linear radius, we use the exact Mattig equation with $q_{0}=1 / 2$ for $(m-M)$ instead of equation (5). Note also that the naive choice of the distance now, i.e., when the light is received, as $D=c z / H_{0}$ was the choice of Hubble in his discussions of the 1930s. It is, in fact, exact for $q_{0}=1$, and it is also exact for all models in the limit of zero redshift, seen directly from the Mattig equations. The Mattig (1958) equation that replaces equation (5), using $q_{0}=1 / 2$ with $H_{0}=50$, is

$$
m-M=5 \log \left\{2\left[1+z-(1+z)^{0.5}\right]\right\}+43.891
$$

(Sandage 1961; 1995, eq. [5.14]). For a redshift of $z=0.05$, the difference between equation (7) and (5) is, in fact, 0.026 mag, as expected from equation (6).

We have used equation (7) to calculate the $m-M$ values listed in the first column of Table 1 . The absolute magnitudes at various $\eta$-values are listed for each cluster in the body of the table using the adopted apparent magnitudes (not listed) at each $\eta$ radius and this calculated $m-M$ modulus. The observed apparent magnitudes (corrected for Galactic absorption and $K$ term) can, therefore, be recovered by applying the $m-M$ value in reverse using the listed absolute magnitude values.

The fourth entry in the first column, $f$, is the log of the number which, when added to log of the observed angular 
TABLE 1

Basic Data From Postman-Lauer on Local First-Ranked Cluster Elliptical GALAXIES (CAPE/COUSINS $R$ BAND; $H_{0}=50$ )

\begin{tabular}{|c|c|c|c|c|c|c|}
\hline \multirow[b]{2}{*}{$\begin{array}{c}\text { CLUUSTER } \\
z \\
m-M \\
f^{\mathrm{a}}\end{array}$} & \multicolumn{6}{|c|}{$\eta$} \\
\hline & $\begin{array}{c}1.0 \\
M(\eta) \\
\log R \\
\langle\mathrm{SB}\rangle\end{array}$ & $\begin{array}{c}1.3 \\
M(\eta) \\
\log R \\
\langle\mathrm{SB}\rangle\end{array}$ & $\begin{array}{c}1.5 \\
M(\eta) \\
\log R \\
\langle\mathrm{SB}\rangle\end{array}$ & $\begin{array}{c}1.7 \\
M(\eta) \\
\log R \\
\langle\mathrm{SB}\rangle\end{array}$ & $\begin{array}{c}2.0 \\
M(\eta) \\
\log R \\
\langle\mathrm{SB}\rangle\end{array}$ & $\begin{array}{c}2.5 \\
M(\eta) \\
\log R \\
\langle\mathrm{SB}\rangle\end{array}$ \\
\hline \multicolumn{7}{|l|}{ A76: } \\
\hline $.0378 \ldots \ldots$ & & -23.37 & -23.79 & -24.11 & & \\
\hline $36.80 \ldots \ldots$ & & 4.192 & 4.510 & 4.770 & & \\
\hline $3.013 \ldots \ldots$ & & 20.57 & 21.75 & 22.73 & & \\
\hline \multicolumn{7}{|l|}{ A119: } \\
\hline $.0450 \ldots \ldots$ & & -24.31 & -24.39 & -24.48 & -24.54 & \\
\hline $37.18 \ldots \ldots$ & & 4.619 & 4.683 & 4.758 & 4.818 & \\
\hline $3.083 \ldots \ldots$ & & 21.79 & 22.03 & 22.31 & 22.56 & \\
\hline \multicolumn{7}{|l|}{ A147: } \\
\hline $.0439 \ldots \ldots$ & & & & -23.53 & -23.76 & \\
\hline $37.12 \ldots \ldots$ & & & & 4.324 & 4.549 & \\
\hline $3.074 \ldots \ldots$ & & & & 21.08 & 22.98 & \\
\hline \multicolumn{7}{|l|}{ A160: } \\
\hline $.0443 \ldots \ldots$ & -23.05 & -23.55 & -23.74 & & & \\
\hline $37.14 \ldots \ldots$ & 4.227 & 4.523 & 4.649 & & & \\
\hline $3.077 \ldots \ldots$ & 21.08 & 22.06 & 22.50 & & & \\
\hline \multicolumn{7}{|l|}{ A168: } \\
\hline $.0453 \ldots \ldots$ & & -23.51 & -23.78 & -24.01 & -24.06 & \\
\hline $37.19 \ldots \ldots$ & & 4.266 & 4.473 & 4.676 & 4.721 & \\
\hline $3.086 \ldots \ldots$ & & 20.82 & 21.59 & 22.37 & 22.55 & \\
\hline \multicolumn{7}{|l|}{ A189: } \\
\hline $.0335 \ldots \ldots$ & & & & $(-22.81)$ & -22.96 & $(-23.14)$ \\
\hline $36.53 \ldots \ldots$ & & & & (4.204) & 4.382 & (4.689) \\
\hline $2.964 \ldots \ldots$ & & & & $(21.16)$ & 21.90 & (23.26) \\
\hline \multicolumn{7}{|l|}{ A193: } \\
\hline $.0491 \ldots \ldots$ & & -24.18 & & & & \\
\hline $37.37 \ldots \ldots$ & & 4.618 & & & & \\
\hline $3.118 \ldots \ldots$ & & 23.93 & & & & \\
\hline \multicolumn{7}{|l|}{ A194: } \\
\hline $.0183 \ldots \ldots$ & & -23.43 & -23.62 & -23.89 & & \\
\hline $35.21 \ldots \ldots$ & & 4.294 & 4.454 & 4.669 & & \\
\hline $2.712 \ldots \ldots$ & & 20.93 & 21.54 & 22.35 & & \\
\hline \multicolumn{7}{|l|}{ A195: } \\
\hline $.0433 \ldots \ldots$ & & & -23.31 & -23.50 & -23.72 & \\
\hline $37.09 \ldots \ldots$ & & & 4.198 & 4.373 & 4.633 & \\
\hline $3.068 \ldots \ldots$ & & & 20.67 & 21.34 & 22.44 & \\
\hline \multicolumn{7}{|l|}{ A260: } \\
\hline $.0374 \ldots \ldots$ & & & -24.22 & & & \\
\hline $36.77 \ldots \ldots$ & & & (4.689) & & & \\
\hline $3.009 \ldots \ldots$ & & & $(22.19)$ & & & \\
\hline \multicolumn{7}{|l|}{ A261: } \\
\hline $.0469 \ldots \ldots$ & & -23.50 & -23.80 & -24.03 & & \\
\hline $37.27 \ldots \ldots$ & & $(4.260)$ & 4.487 & (4.685) & & \\
\hline $3.100 \ldots \ldots$ & & $(20.81)$ & 21.65 & $(22.41)$ & & \\
\hline \multicolumn{7}{|l|}{ A262: } \\
\hline $.0171 \ldots \ldots$ & & & & & $(-23.68)$ & \\
\hline $35.06 \ldots \ldots$ & & & & & (4.634) & \\
\hline $2.684 \ldots \ldots$ & & & & & $(22.37)$ & \\
\hline \multicolumn{7}{|l|}{ A347: } \\
\hline $.0194 \ldots \ldots$ & & -23.23 & -23.72 & $(-23.90)$ & & \\
\hline $35.34 \ldots \ldots$ & & 4.257 & 4.617 & (4.787) & & \\
\hline $2.737 \ldots \ldots$ & & 20.95 & 22.26 & $(22.93)$ & & \\
\hline A376: & & & & & & \\
\hline $.0496 \ldots \ldots$ & & -23.91 & -24.23 & & & \\
\hline $37.39 \ldots \ldots$ & & 4.531 & (4.777) & & & \\
\hline $3.122 \ldots \ldots$ & & 21.77 & $(22.68)$ & & & \\
\hline A397: & & & & & & \\
\hline $.0336 \ldots \ldots$ & & -23.32 & -23.92 & & & \\
\hline $36.54 \ldots \ldots$ & & 4.170 & 4.615 & & & \\
\hline $2.965 \ldots \ldots$ & & 20.49 & 22.11 & & & \\
\hline
\end{tabular}


TABLE 1-Continued

\begin{tabular}{|c|c|c|c|c|c|c|}
\hline \multirow{5}{*}{$\begin{array}{c}\text { CLUSTER } \\
z \\
m-M \\
f^{\mathrm{a}}\end{array}$} & \multicolumn{6}{|c|}{$\eta$} \\
\hline & 1.0 & 1.3 & 1.5 & 1.7 & 2.0 & 2.5 \\
\hline & $M(\eta)$ & $M(\eta)$ & $M(\eta)$ & $M(\eta)$ & $M(\eta)$ & $M(\eta)$ \\
\hline & $\log R$ & $\log R$ & $\log R$ & $\log R$ & $\log R$ & $\log R$ \\
\hline & $\langle\mathrm{SB}\rangle$ & $\langle\mathrm{SB}\rangle$ & $\langle\mathrm{SB}\rangle$ & $\langle\mathrm{SB}\rangle$ & $\langle\mathrm{SB}\rangle$ & $\langle\mathrm{SB}\rangle$ \\
\hline \multicolumn{7}{|l|}{ A407: } \\
\hline $.0477 \ldots \ldots$ & $(-23.43)$ & -23.91 & -23.95 & -24.02 & & \\
\hline $37.31 \ldots \ldots$ & 4.342 & 4.607 & 4.649 & 4.693 & & \\
\hline $3.107 \ldots \ldots$ & $(21.30)$ & 22.14 & 22.31 & 22.46 & & \\
\hline \multicolumn{7}{|l|}{ A419: } \\
\hline $.0408 \ldots \ldots$ & & & & & -22.80 & -22.97 \\
\hline $36.96 \ldots \ldots$ & & & & & 4.356 & 4.584 \\
\hline $3.044 \ldots \ldots$ & & & & & 21.96 & 22.93 \\
\hline \multicolumn{7}{|l|}{ A496: } \\
\hline $.0327 \ldots \ldots$ & & -24.10 & -24.22 & -24.42 & & \\
\hline $36.48 \ldots \ldots$ & & 4.594 & 4.671 & 4.829 & & \\
\hline $2.954 \ldots \ldots$ & & 21.82 & 22.09 & 22.68 & & \\
\hline \multicolumn{7}{|l|}{ A533: } \\
\hline $.0467 \ldots \ldots$ & & & -23.39 & $(-23.72)$ & -24.05 & \\
\hline $37.26 \ldots \ldots$ & & & 4.298 & $(4.598)$ & 4.948 & \\
\hline $3.098 \ldots \ldots$ & & & 21.11 & $(22.28)$ & 23.70 & \\
\hline \multicolumn{7}{|l|}{ A539: } \\
\hline $.0291 \ldots \ldots$ & & & -23.40 & -23.59 & -23.69 & \\
\hline $36.22 \ldots \ldots$ & & & 4.311 & 4.488 & 4.594 & \\
\hline $2.906 \ldots \ldots$ & & & 21.09 & 21.78 & 22.21 & \\
\hline \multicolumn{7}{|l|}{ A548: } \\
\hline $.0393 \ldots \ldots$ & & & -23.50 & -23.72 & -23.96 & \\
\hline $36.88 \ldots \ldots$ & & & 4.329 & 4.549 & 4.789 & \\
\hline $3.029 \ldots \ldots$ & & & 21.12 & 22.00 & 22.96 & \\
\hline \multicolumn{7}{|l|}{ A569: } \\
\hline $.0194 \ldots \ldots$ & & & $(-23.35)$ & -23.46 & $(-23.71)$ & \\
\hline $35.34 \ldots \ldots$ & & & 4.279 & 4.374 & (4.697) & \\
\hline $2.737 \ldots \ldots$ & & & $(20.94)$ & 21.31 & $(22.67)$ & \\
\hline \multicolumn{7}{|l|}{ A576: } \\
\hline $.0388 \ldots \ldots$ & & & & & -23.07 & -23.16 \\
\hline $36.86 \ldots \ldots$ & & & & & 4.267 & 4.404 \\
\hline $3.024 \ldots \ldots$ & & & & & 21.25 & 21.84 \\
\hline \multicolumn{7}{|l|}{ A634: } \\
\hline $.0275 \ldots \ldots$ & & & -23.24 & -23.48 & & \\
\hline $36.10 \ldots \ldots$ & & & 4.282 & 4.482 & & \\
\hline $2.882 \ldots \ldots$ & & & 21.10 & 21.86 & & \\
\hline \multicolumn{7}{|l|}{ A671: } \\
\hline $.0506 \ldots \ldots$ & & -24.53 & -24.76 & -24.88 & -24.97 & \\
\hline $37.44 \ldots \ldots$ & & 4.643 & 4.830 & 4.940 & $(5.080)$ & \\
\hline $3.130 \ldots \ldots$ & & 21.72 & 22.42 & 22.85 & $(23.46)$ & \\
\hline \multicolumn{7}{|l|}{ A779: } \\
\hline $.0225 \ldots \ldots$ & & $(-23.71)$ & -24.51 & $(-24.68)$ & & \\
\hline $35.66 \ldots \ldots$ & & (4.129) & 4.691 & 4.849 & & \\
\hline $2.799 \ldots \ldots$ & & $(19.84)$ & 21.85 & $(22.47)$ & & \\
\hline A912: & & & & & & \\
\hline $.0446 \ldots \ldots$ & & & & -22.92 & -23.14 & -23.28 \\
\hline $37.16 \ldots \ldots$ & & & & 4.295 & 4.545 & 4.762 \\
\hline $3.080 \ldots \ldots$ & & & & 21.56 & 22.59 & 23.53 \\
\hline A957: & & & & & & \\
\hline $.0441 \ldots \ldots$ & & -24.53 & -24.78 & -24.91 & -25.10 & \\
\hline $37.13 \ldots \ldots$ & & 4.697 & 4.875 & 5.011 & 5.198 & \\
\hline $3.075 \ldots \ldots$ & & 21.95 & 22.59 & 23.14 & 23.89 & \\
\hline A999: & & & & & & \\
\hline $.0316 \ldots \ldots$ & & & & -23.49 & -23.61 & \\
\hline $36.40 \ldots \ldots$ & & & & 4.516 & 4.665 & \\
\hline $2.940 \ldots \ldots$ & & & & 22.03 & 22.66 & \\
\hline A1016: & & & & & & \\
\hline $.0317 \ldots \ldots$ & & & & -23.08 & $(-23.46)$ & \\
\hline $36.41 \ldots \ldots$ & & & & 4.361 & $(4.781)$ & \\
\hline $2.941 \ldots \ldots$ & & & & 21.67 & $(23.39)$ & \\
\hline
\end{tabular}


TABLE 1-Continued

\begin{tabular}{|c|c|c|c|c|c|c|}
\hline \multirow{5}{*}{$\begin{array}{c}\text { CLUSTER } \\
z \\
m-M \\
f^{\text {a }}\end{array}$} & \multicolumn{6}{|c|}{$\eta$} \\
\hline & 1.0 & 1.3 & 1.5 & 1.7 & 2.0 & 2.5 \\
\hline & $M(\eta)$ & $M(\eta)$ & $M(\eta)$ & $M(\eta)$ & $M(\eta)$ & $M(\eta)$ \\
\hline & $\log R$ & $\log R$ & $\log R$ & $\log R$ & $\log R$ & $\log R$ \\
\hline & $\langle\mathrm{SB}\rangle$ & $\langle\mathrm{SB}\rangle$ & $\langle\mathrm{SB}\rangle$ & $\langle\mathrm{SB}\rangle$ & $\langle\mathrm{SB}\rangle$ & $\langle\mathrm{SB}\rangle$ \\
\hline \multicolumn{7}{|l|}{ A1060: } \\
\hline $.0115 \ldots \ldots$ & -23.20 & -24.16 & & & & \\
\hline $34.20 \ldots \ldots$ & 4.302 & 4.841 & & & & \\
\hline $2.516 \ldots \ldots$ & 21.17 & 22.91 & & & & \\
\hline \multicolumn{7}{|l|}{ A1100: } \\
\hline $.0463 \ldots \ldots$ & & -23.30 & -23.58 & -23.91 & & \\
\hline $37.24 \ldots \ldots$ & & 4.230 & 4.435 & 4.735 & & \\
\hline $3.095 \ldots \ldots$ & & 20.86 & 21.60 & 22.77 & & \\
\hline \multicolumn{7}{|l|}{ A1139: } \\
\hline $.0387 \ldots \ldots$ & & $(-23.13)$ & -23.87 & & & \\
\hline $36.85 \ldots \ldots$ & & 4.171 & 4.708 & & & \\
\hline $3.023 \ldots \ldots$ & & $(20.70)$ & 22.65 & & & \\
\hline \multicolumn{7}{|l|}{ A1142: } \\
\hline $.0345 \ldots \ldots$ & & & -23.76 & -23.96 & & \\
\hline $36.60 \ldots \ldots$ & & & 4.633 & 4.833 & & \\
\hline $2.976 \ldots \ldots$ & & & 22.37 & 23.17 & & \\
\hline \multicolumn{7}{|l|}{ A1177: } \\
\hline $.0315 \ldots \ldots$ & & -23.94 & -24.35 & & & \\
\hline $36.40 \ldots \ldots$ & & 4.575 & 4.873 & & & \\
\hline $2.938 \ldots \ldots$ & & 21.89 & 22.97 & & & \\
\hline \multicolumn{7}{|l|}{ A1185: } \\
\hline $.0329 \ldots \ldots$ & & -23.49 & -23.64 & & & \\
\hline $36.49 \ldots \ldots$ & & 4.303 & 4.431 & & & \\
\hline $2.956 \ldots \ldots$ & & 20.98 & 21.47 & & & \\
\hline \multicolumn{7}{|l|}{ A1213: } \\
\hline $.0467 \ldots \ldots$ & & & -23.45 & -23.60 & -23.80 & \\
\hline $37.26 \ldots \ldots$ & & & 4.285 & 4.398 & 4.606 & \\
\hline $3.098 \ldots \ldots$ & & & 20.99 & 21.40 & 22.24 & \\
\hline \multicolumn{7}{|l|}{ A1228: } \\
\hline $.0365 \ldots \ldots$ & & & -23.03 & -23.20 & & \\
\hline $36.72 \ldots \ldots$ & & & 4.161 & 4.373 & & \\
\hline $2.999 \ldots \ldots$ & & & 20.74 & 21.63 & & \\
\hline \multicolumn{7}{|l|}{ A1257: } \\
\hline $.0343 \ldots \ldots$ & & & & & -22.52 & -22.86 \\
\hline $36.58 \ldots \ldots$ & & & & & 4.193 & 4.448 \\
\hline $2.973 \ldots \ldots$ & & & & & 21.40 & 22.34 \\
\hline \multicolumn{7}{|l|}{ A1267: } \\
\hline $.0326 \ldots \ldots$ & & & & & -23.11 & -23.31 \\
\hline $36.47 \ldots \ldots$ & & & & & 4.253 & 4.568 \\
\hline $2.953 \ldots \ldots$ & & & & & 21.10 & 22.48 \\
\hline \multicolumn{7}{|l|}{ A1308: } \\
\hline $.0511 \ldots \ldots$ & & $(-23.58)$ & -24.10 & -24.33 & -24.55 & \\
\hline $37.46 \ldots \ldots$ & & 4.164 & 4.564 & 4.759 & 4.974 & \\
\hline $3.134 \ldots \ldots$ & & $(20.27)$ & 21.75 & 22.50 & 23.35 & \\
\hline A1314: & & & & & & \\
\hline $.0330 \ldots \ldots$ & & $(-23.42)$ & -23.91 & -24.19 & -24.44 & \\
\hline $36.50 \ldots \ldots$ & & 4.218 & 4.578 & 4.800 & (5.058) & \\
\hline $2.958 \ldots \ldots$ & & $(20.62)$ & 21.93 & 22.76 & $(23.80)$ & \\
\hline A1367: & & & & & & \\
\hline $.0213 \ldots \ldots$ & & $(-23.34)$ & -23.65 & -23.85 & -24.01 & \\
\hline $35.54 \ldots \ldots$ & & 4.156 & 4.368 & 4.536 & 4.746 & \\
\hline $2.776 \ldots \ldots$ & & $(20.34)$ & 21.09 & 21.73 & 22.62 & \\
\hline A1631: & & & & & & \\
\hline $.0461 \ldots \ldots$ & & -23.70 & -24.07 & $(-24.43)$ & & \\
\hline $37.23 \ldots \ldots$ & & 4.363 & 4.633 & 5.013 & & \\
\hline $3.093 \ldots \ldots$ & & 21.12 & 22.10 & (23.64) & & \\
\hline A1644: & & & & & & \\
\hline $.0468 \ldots \ldots$ & -24.25 & -24.73 & $(-24.92)$ & & & \\
\hline $37.27 \ldots \ldots$ & 4.539 & 4.824 & 4.974 & & & \\
\hline $3.099 \ldots \ldots$ & 21.46 & 22.41 & $(22.97)$ & & & \\
\hline
\end{tabular}


TABLE 1-Continued

\begin{tabular}{|c|c|c|c|c|c|c|}
\hline \multirow{5}{*}{$\begin{array}{c}\text { CLUSTER } \\
z \\
m-M \\
f^{\text {a }}\end{array}$} & \multicolumn{6}{|c|}{$\eta$} \\
\hline & 1.0 & 1.3 & 1.5 & 1.7 & 2.0 & 2.5 \\
\hline & $M(\eta)$ & $M(\eta)$ & $M(\eta)$ & $M(\eta)$ & $M(\eta)$ & $M(\eta)$ \\
\hline & $\log R$ & $\log R$ & $\log R$ & $\log R$ & $\log R$ & $\log R$ \\
\hline & $\langle\mathrm{SB}\rangle$ & $\langle\mathrm{SB}\rangle$ & $\langle\mathrm{SB}\rangle$ & $\langle\mathrm{SB}\rangle$ & $\langle\mathrm{SB}\rangle$ & $\langle\mathrm{SB}\rangle$ \\
\hline \multicolumn{7}{|l|}{ A1656: } \\
\hline $.0232 \ldots \ldots$ & & -24.00 & -24.48 & $(-24.78)$ & & \\
\hline $35.73 \ldots \ldots$ & & 4.262 & 4.612 & 4.887 & & \\
\hline $2.812 \ldots \ldots$ & & 20.22 & 21.49 & $(22.57)$ & & \\
\hline \multicolumn{7}{|l|}{ A1736: } \\
\hline $.0446 \ldots \ldots$ & & -24.03 & -24.45 & -24.76 & $(-24.95)$ & \\
\hline $37.16 \ldots \ldots$ & & 4.230 & 4.540 & 4.830 & $(5.060)$ & \\
\hline $3.080 \ldots \ldots$ & & 20.12 & 21.25 & 22.39 & $(23.35)$ & \\
\hline \multicolumn{7}{|l|}{ A1836: } \\
\hline $.0363 \ldots \ldots$ & & -23.62 & -23.86 & -24.05 & -24.26 & \\
\hline $36.71 \ldots \ldots$ & & 4.247 & 4.445 & 4.589 & 4.827 & \\
\hline $2.997 \ldots \ldots$ & & 20.58 & 21.33 & 21.86 & 22.84 & \\
\hline \multicolumn{7}{|l|}{ A1983: } \\
\hline $.0455 \ldots \ldots$ & & & & -23.18 & -23.35 & -23.47 \\
\hline $37.20 \ldots \ldots$ & & & & 4.156 & 4.343 & 4.538 \\
\hline $3.088 \ldots \ldots$ & & & & 20.60 & 21.37 & 22.22 \\
\hline \multicolumn{7}{|l|}{ A2040: } \\
\hline $.0457 \ldots \ldots$ & -23.61 & -23.89 & -23.99 & -24.04 & -24.10 & \\
\hline $37.21 \ldots \ldots$ & 4.540 & 4.685 & 4.750 & 4.790 & 4.860 & \\
\hline $3.090 \ldots \ldots$ & 22.09 & 22.54 & 22.76 & 22.91 & 23.20 & \\
\hline \multicolumn{7}{|l|}{ A2052: } \\
\hline $.0352 \ldots \ldots$ & -23.85 & -24.55 & $(-24.77)$ & & & \\
\hline $36.64 \ldots \ldots$ & 4.434 & 4.824 & $(4.974)$ & & & \\
\hline $2.984 \ldots \ldots$ & 21.28 & 22.53 & $(23.06)$ & & & \\
\hline \multicolumn{7}{|l|}{ A2063: } \\
\hline $.0355 \ldots \ldots$ & -23.34 & -23.92 & -24.24 & -24.41 & & \\
\hline $36.66 \ldots \ldots$ & 4.262 & 4.602 & 4.837 & 4.987 & & \\
\hline $2.987 \ldots \ldots$ & 20.94 & 22.06 & 22.91 & 23.49 & & \\
\hline \multicolumn{7}{|l|}{ A2107: } \\
\hline $.0419 \ldots \ldots$ & -23.77 & -24.38 & -24.68 & & & \\
\hline $37.02 \ldots \ldots$ & 4.265 & 4.585 & 4.815 & & & \\
\hline $3.055 \ldots \ldots$ & 20.54 & 21.53 & 22.38 & & & \\
\hline \multicolumn{7}{|l|}{ A2147: } \\
\hline $.0353 \ldots \ldots$ & & -23.60 & -24.02 & -24.32 & & \\
\hline $36.65 \ldots \ldots$ & & 4.435 & 4.755 & 5.005 & & \\
\hline $2.985 \ldots \ldots$ & & 21.54 & 22.72 & 23.67 & & \\
\hline \multicolumn{7}{|l|}{ A2151: } \\
\hline $.0373 \ldots \ldots$ & & -23.85 & -24.06 & -24.21 & -24.32 & \\
\hline $36.77 \ldots \ldots$ & & 4.550 & 4.708 & 4.818 & 4.923 & \\
\hline $3.008 \ldots \ldots$ & & 21.87 & 22.45 & 22.85 & 23.27 & \\
\hline \multicolumn{7}{|l|}{ A2152: } \\
\hline $.0456 \ldots \ldots$ & & & -23.31 & -23.55 & -23.75 & \\
\hline $37.21 \ldots \ldots$ & & & 4.189 & 4.449 & 4.744 & \\
\hline $3.089 \ldots \ldots$ & & & 20.64 & 21.70 & 22.98 & \\
\hline A2162: & & & & & & \\
\hline $.0325 \ldots \ldots$ & & & -23.71 & -24.06 & $(-24.44)$ & \\
\hline $36.47 \ldots \ldots$ & & & 4.381 & 4.671 & $(5.101)$ & \\
\hline $2.951 \ldots \ldots$ & & & 21.15 & 22.25 & $(24.02)$ & \\
\hline A2197: & & & & & & \\
\hline $.0307 \ldots \ldots$ & & -24.01 & -24.33 & -24.63 & $(-24.97)$ & \\
\hline $36.34 \ldots \ldots$ & & 4.273 & 4.513 & 4.778 & $(5.228)$ & \\
\hline $2.928 \ldots \ldots$ & & 20.30 & 21.18 & 22.20 & $(24.11)$ & \\
\hline A2199: & & & & & & \\
\hline $.0307 \ldots \ldots$ & $(-23.71)$ & -24.45 & -24.82 & & & \\
\hline $36.34 \ldots \ldots$ & 4.258 & 4.698 & 4.988 & & & \\
\hline $2.928 \ldots \ldots$ & $(20.52)$ & 21.98 & 23.06 & & & \\
\hline A2247: & & & & & & \\
\hline $.0396 \ldots \ldots$ & & & $(-23.10)$ & -23.30 & -23.58 & \\
\hline $36.90 \ldots \ldots$ & & & $(4.082)$ & 4.267 & 4.572 & \\
\hline $3.032 \ldots \ldots$ & & & $(20.29)$ & 21.02 & 22.26 & \\
\hline
\end{tabular}


TABLE 1-Continued

\begin{tabular}{|c|c|c|c|c|c|c|}
\hline \multirow[b]{2}{*}{$\begin{array}{c}\text { CLUSTER } \\
z \\
m-M \\
f^{\text {a }}\end{array}$} & \multicolumn{6}{|c|}{$\eta$} \\
\hline & $\begin{array}{c}1.0 \\
M(\eta) \\
\log R \\
\langle\mathrm{SB}\rangle\end{array}$ & $\begin{array}{c}1.3 \\
M(\eta) \\
\log R \\
\langle\mathrm{SB}\rangle\end{array}$ & $\begin{array}{c}1.5 \\
M(\eta) \\
\log R \\
\langle\mathrm{SB}\rangle\end{array}$ & $\begin{array}{c}1.7 \\
M(\eta) \\
\log R \\
\langle\mathrm{SB}\rangle\end{array}$ & $\begin{array}{c}2.0 \\
M(\eta) \\
\log R \\
\langle\mathrm{SB}\rangle\end{array}$ & $\begin{array}{c}2.5 \\
M(\eta) \\
\log R \\
\langle\mathrm{SB}\rangle\end{array}$ \\
\hline A2572: & & & & & & \\
\hline $\begin{array}{l}.0423 \ldots \ldots \\
37.04 \ldots \ldots \\
3.059 \ldots \ldots\end{array}$ & & & $\begin{array}{c}(-23.76 \\
(4.409) \\
(21.27)\end{array}$ & $\begin{array}{c}(-24.05) \\
(4.644) \\
(22.16)\end{array}$ & $\begin{array}{c}(-24.26) \\
(4.909) \\
(23.27)\end{array}$ & \\
\hline A2589: & & & & & & \\
\hline $\begin{array}{l}.0422 \ldots \ldots \\
37.04 \ldots \ldots \\
3.058 \ldots \ldots\end{array}$ & $\begin{array}{c}-23.47 \\
4.304 \\
21.04\end{array}$ & $\begin{array}{c}-24.15 \\
4.698 \\
22.33\end{array}$ & $\begin{array}{c}(-24.37) \\
(4.898) \\
(23.11)\end{array}$ & & & \\
\hline A2593: & & & & & & \\
\hline $\begin{array}{l}.0424 \ldots \ldots \\
37.05 \ldots \ldots \\
3.060 \ldots \ldots\end{array}$ & $\begin{array}{c}-23.55 \\
4.308 \\
20.98\end{array}$ & $\begin{array}{c}-23.92 \\
4.520 \\
21.67\end{array}$ & $\begin{array}{c}(-24.13) \\
(4.680) \\
(22.26)\end{array}$ & & & \\
\hline $\begin{array}{r}\mathrm{A} 2634: \\
.0314 \ldots \ldots \\
36.39 \ldots \ldots \\
2.937 \ldots \ldots\end{array}$ & & $\begin{array}{c}(-24.13) \\
(4.517) \\
(21.40)\end{array}$ & $\begin{array}{c}(-24.51) \\
(4.797) \\
(22.42)\end{array}$ & & & \\
\hline $\begin{array}{r}\mathrm{A} 2657: \\
\quad .0411 \ldots \ldots \\
36.98 \ldots \ldots \\
3.047 \ldots \ldots\end{array}$ & & & $\begin{array}{c}(-22.65) \\
(3.967) \\
(20.17)\end{array}$ & $\begin{array}{c}(-22.83) \\
(4.097) \\
(20.64)\end{array}$ & $\begin{array}{c}-23.04 \\
4.317 \\
21.53\end{array}$ & $\begin{array}{c}(-23.25) \\
(4.647) \\
(22.97)\end{array}$ \\
\hline $\begin{array}{l}\text { A2666: } \\
.0277 \ldots \ldots \\
36.12 \ldots \ldots \\
2.885 \ldots \ldots\end{array}$ & & $\begin{array}{c}(-23.54) \\
(4.105) \\
(19.92)\end{array}$ & $\begin{array}{c}-23.91 \\
4.370 \\
20.88\end{array}$ & $\begin{array}{c}-24.04 \\
4.495 \\
21.37\end{array}$ & $\begin{array}{c}(-24.19) \\
4.670 \\
(22.10)\end{array}$ & \\
\hline $\begin{array}{r}\text { A2717: } \\
.0492 \ldots \ldots \\
37.38 \ldots \ldots \\
3.119 \ldots \ldots\end{array}$ & $\begin{array}{c}(-24.07 \\
(4.629) \\
(22.10)\end{array}$ & $\begin{array}{c}(-24.53) \\
(4.869) \\
(22.84)\end{array}$ & $\begin{array}{c}(-24.83) \\
(5.089) \\
(23.64)\end{array}$ & & & \\
\hline $\begin{array}{l}\mathrm{A} 2731: \\
.0312 \ldots \ldots \\
36.38 \ldots \ldots \\
2.935 \ldots \ldots\end{array}$ & & & $\begin{array}{c}-23.76 \\
4.515 \\
21.76\end{array}$ & $\begin{array}{c}-23.93 \\
4.695 \\
22.49\end{array}$ & $\begin{array}{c}(-24.10) \\
(4.895) \\
(23.32)\end{array}$ & \\
\hline $\begin{array}{l}\mathrm{A} 2806: \\
.0272 \ldots \ldots \\
36.08 \ldots \ldots \\
2.878 \ldots \ldots\end{array}$ & & & & $\begin{array}{c}(-23.31) \\
4.255 \\
(20.90)\end{array}$ & $\begin{array}{c}-23.51 \\
4.478 \\
21.81\end{array}$ & $\begin{array}{r}(-23.69) \\
4.788 \\
(23.18)\end{array}$ \\
\hline $\begin{array}{r}\mathrm{A} 2870: \\
.0239 \ldots \ldots \\
35.79 \ldots \ldots \\
2.824 \ldots \ldots\end{array}$ & & $\begin{array}{c}-23.62 \\
4.349 \\
21.04\end{array}$ & $\begin{array}{c}-23.92 \\
4.554 \\
21.76\end{array}$ & $\begin{array}{c}(-24.10) \\
(4.711) \\
(22.37)\end{array}$ & & \\
\hline $\begin{array}{r}\mathrm{A} 2877: \\
.0242 \ldots \ldots \\
35.82 \ldots \ldots \\
2.829 \ldots \ldots\end{array}$ & & $\begin{array}{c}-24.23 \\
4.309 \\
20.23\end{array}$ & $\begin{array}{c}-24.65 \\
4.617 \\
21.35\end{array}$ & $\begin{array}{c}(-24.83) \\
(4.769) \\
(21.93)\end{array}$ & & \\
\hline $\begin{array}{l}\mathrm{A} 2881: \\
\quad .0446 \ldots \ldots \\
37.16 \ldots \ldots \\
3.080 \ldots \ldots\end{array}$ & & & $\begin{array}{c}(-22.70) \\
(4.140) \\
(21.00)\end{array}$ & $\begin{array}{c}-22.90 \\
4.240 \\
21.30\end{array}$ & $\begin{array}{c}-23.03 \\
4.410 \\
22.02\end{array}$ & $\begin{array}{c}(-23.20) \\
(4.680) \\
(23.20)\end{array}$ \\
\hline $\begin{array}{r}\text { A2869: } \\
.0318 \ldots \ldots \\
36.42 \ldots \ldots \\
2.942 \ldots \ldots\end{array}$ & & & & $\begin{array}{c}(-23.03) \\
(4.127) \\
(20.56)\end{array}$ & $\begin{array}{c}-23.25 \\
4.397 \\
21.69\end{array}$ & $\begin{array}{c}(-23.48) \\
(4.752) \\
(23.23)\end{array}$ \\
\hline $\begin{array}{l}\text { A2911: } \\
.0201 \ldots \ldots \\
35.42 \ldots \ldots \\
2.752 \ldots \ldots\end{array}$ & & & $\begin{array}{c}(-22.35) \\
(4.052) \\
(20.81)\end{array}$ & $\begin{array}{c}(-22.51) \\
4.146 \\
(21.12)\end{array}$ & $\begin{array}{c}-22.65 \\
4.297 \\
21.74\end{array}$ & $\begin{array}{c}-22.78 \\
4.484 \\
22.54\end{array}$ \\
\hline $\begin{array}{l}\text { A3144: } \\
\quad .0446 \ldots \ldots \\
37.16 \ldots \ldots \\
3.080 \ldots \ldots\end{array}$ & & & $\begin{array}{r}(-22.91) \\
4.150 \\
(20.84)\end{array}$ & $\begin{array}{c}-23.21 \\
4.372 \\
21.65\end{array}$ & $\begin{array}{c}-23.43 \\
4.630 \\
22.72\end{array}$ & $\begin{array}{c}(-23.53) \\
(4.780) \\
(23.37)\end{array}$ \\
\hline
\end{tabular}


TABLE 1-Continued

\begin{tabular}{|c|c|c|c|c|c|c|}
\hline \multirow{5}{*}{$\begin{array}{c}\text { CLUSTER } \\
z \\
m-M \\
f^{\mathrm{a}}\end{array}$} & \multicolumn{6}{|c|}{$\eta$} \\
\hline & 1.0 & 1.3 & 1.5 & 1.7 & 2.0 & 2.5 \\
\hline & $M(\eta)$ & $M(\eta)$ & $M(\eta)$ & $M(\eta)$ & $M(\eta)$ & $M(\eta)$ \\
\hline & $\log R$ & $\log R$ & $\log R$ & $\log R$ & $\log R$ & $\log R$ \\
\hline & $\langle\mathrm{SB}\rangle$ & $\langle\mathrm{SB}\rangle$ & $\langle\mathrm{SB}\rangle$ & $\langle\mathrm{SB}\rangle$ & $\langle\mathrm{SB}\rangle$ & $\langle\mathrm{SB}\rangle$ \\
\hline \multicolumn{7}{|l|}{ A3193: } \\
\hline $.0339 \ldots \ldots$ & & -23.07 & -23.30 & -23.52 & -23.72 & $(-23.89)$ \\
\hline $36.56 \ldots \ldots$ & & 4.052 & 4.251 & 4.469 & 4.679 & (4.834) \\
\hline $2.969 \ldots \ldots$ & & 20.15 & 20.91 & 21.78 & 22.63 & (23.24) \\
\hline \multicolumn{7}{|l|}{ A3367: } \\
\hline $.0443 \ldots \ldots$ & & & & $(-22.84)$ & -23.19 & \\
\hline $37.14 \ldots \ldots$ & & & & (4.197) & 4.577 & \\
\hline $3.077 \ldots \ldots$ & & & & $(21.14)$ & 22.69 & \\
\hline \multicolumn{7}{|l|}{ A3374: } \\
\hline $.0471 \ldots \ldots$ & & & & & -22.98 & -23.08 \\
\hline $37.28 \ldots \ldots$ & & & & & 4.227 & 4.352 \\
\hline $3.102 \ldots \ldots$ & & & & & 21.17 & 21.69 \\
\hline \multicolumn{7}{|l|}{ A3376: } \\
\hline $.0456 \ldots \ldots$ & & -23.65 & -24.03 & -24.24 & $(-24.43)$ & \\
\hline $37.21 \ldots \ldots$ & & 4.309 & 4.589 & 4.769 & (5.049) & \\
\hline $3.089 \ldots \ldots$ & & 20.90 & 21.92 & 22.61 & $(23.82)$ & \\
\hline \multicolumn{7}{|l|}{ A3381: } \\
\hline $.0375 \ldots \ldots$ & & -22.92 & -23.17 & -23.34 & -23.52 & \\
\hline $36.78 \ldots \ldots$ & & 4.260 & 4.460 & 4.615 & 4.785 & \\
\hline $3.010 \ldots \ldots$ & & 21.35 & 22.10 & 22.71 & 23.38 & \\
\hline \multicolumn{7}{|l|}{ A3389: } \\
\hline $.0262 \ldots \ldots$ & & -23.42 & -23.63 & -23.82 & -24.04 & \\
\hline $36.00 \ldots \ldots$ & & 4.162 & 4.302 & 4.449 & (4.782) & \\
\hline $2.862 \ldots \ldots$ & & 20.32 & 20.81 & 21.36 & $(22.80)$ & \\
\hline \multicolumn{7}{|l|}{ A3395: } \\
\hline $.0482 \ldots \ldots$ & -23.41 & -24.23 & & & & \\
\hline $37.33 \ldots \ldots$ & 4.271 & 4.771 & & & & \\
\hline $3.111 \ldots \ldots$ & 20.96 & 22.64 & & & & \\
\hline \multicolumn{7}{|l|}{ A3526: } \\
\hline $.0107 \ldots \ldots$ & & -24.17 & -24.37 & $(-24.50)$ & & \\
\hline $34.04 \ldots \ldots$ & & 4.455 & 4.610 & (4.733) & & \\
\hline $2.485 \ldots \ldots$ & & 20.96 & 21.54 & $(22.02)$ & & \\
\hline \multicolumn{7}{|l|}{ A3528: } \\
\hline $.0536 \ldots \ldots$ & & -24.49 & -24.95 & $(-25.21)$ & & \\
\hline $37.56 \ldots \ldots$ & & 4.635 & 4.985 & $(5.218)$ & & \\
\hline $3.153 \ldots \ldots$ & & 21.72 & 23.01 & $(23.92)$ & & \\
\hline \multicolumn{7}{|l|}{ A3530: } \\
\hline $.0533 \ldots \ldots$ & & -24.86 & $(-25.03)$ & & & \\
\hline $37.55 \ldots \ldots$ & & 4.851 & 4.978 & & & \\
\hline $3.151 \ldots \ldots$ & & 22.43 & (22.90) & & & \\
\hline \multicolumn{7}{|l|}{ A3532: } \\
\hline $.0547 \ldots \ldots$ & & -24.96 & & & & \\
\hline $37.61 \ldots \ldots$ & & 5.011 & & & & \\
\hline $3.161 \ldots \ldots$ & & 23.14 & & & & \\
\hline A3537: & & & & & & \\
\hline $.0161 \ldots \ldots$ & & & -23.58 & -24.08 & & \\
\hline $34.93 \ldots \ldots$ & & & 4.296 & 4.737 & & \\
\hline $2.658 \ldots \ldots$ & & & 20.78 & 22.49 & & \\
\hline A3542: & & & & & & \\
\hline $.0339 \ldots \ldots$ & & & & & $(-22.90)$ & $(-23.15)$ \\
\hline $36.56 \ldots \ldots$ & & & & & (4.189) & (4.546) \\
\hline $2.969 \ldots \ldots$ & & & & & $(21.00)$ & $(22.54)$ \\
\hline A3553: & & & & & & \\
\hline $.0474 \ldots \ldots$ & & & & -22.92 & -23.13 & $(-23.27)$ \\
\hline $37.29 \ldots \ldots$ & & & & 4.304 & 4.529 & (4.754) \\
\hline $3.104 \ldots \ldots$ & & & & 21.61 & 22.53 & $(23.51)$ \\
\hline A3554: & & & & & & \\
\hline $.0470 \ldots \ldots$ & & -23.74 & -24.05 & -24.25 & $(-24.43)$ & \\
\hline $37.27 \ldots \ldots$ & & 4.506 & 4.733 & 4.909 & $(5.074)$ & \\
\hline $3.101 \ldots \ldots$ & & 21.80 & 22.62 & 23.30 & $(23.94)$ & \\
\hline
\end{tabular}


TABLE 1-Continued

\begin{tabular}{|c|c|c|c|c|c|c|}
\hline \multirow{5}{*}{$\begin{array}{c}\text { CLUSTER } \\
z \\
m-M \\
f^{\mathrm{a}}\end{array}$} & \multicolumn{6}{|c|}{$\eta$} \\
\hline & 1.0 & 1.3 & 1.5 & 1.7 & 2.0 & 2.5 \\
\hline & $M(\eta)$ & $M(\eta)$ & $M(\eta)$ & $M(\eta)$ & $M(\eta)$ & $M(\eta)$ \\
\hline & $\log R$ & $\log R$ & $\log R$ & $\log R$ & $\log R$ & $\log R$ \\
\hline & $\langle\mathrm{SB}\rangle$ & $\langle\mathrm{SB}\rangle$ & $\langle\mathrm{SB}\rangle$ & $\langle\mathrm{SB}\rangle$ & $\langle\mathrm{SB}\rangle$ & $\langle\mathrm{SB}\rangle$ \\
\hline \multicolumn{7}{|l|}{ A3556: } \\
\hline $.0476 \ldots \ldots$ & & -23.94 & -24.25 & -24.42 & -24.54 & \\
\hline $37.30 \ldots \ldots$ & & 4.252 & 4.501 & 4.644 & 4.806 & \\
\hline $3.106 \ldots \ldots$ & & 20.33 & 21.27 & 21.81 & 22.50 & \\
\hline \multicolumn{7}{|l|}{ A5358: } \\
\hline $.0470 \ldots \ldots$ & -24.40 & -25.04 & -25.39 & & & \\
\hline $37.27 \ldots \ldots$ & 4.451 & 4.827 & 5.081 & & & \\
\hline $3.101 \ldots \ldots$ & 20.86 & 22.10 & 23.02 & & & \\
\hline \multicolumn{7}{|l|}{ A3559: } \\
\hline $.0467 \ldots \ldots$ & & $(-23.83)$ & -24.44 & -24.62 & -24.74 & \\
\hline $37.26 \ldots \ldots$ & & 4.243 & 4.643 & 4.798 & 4.958 & \\
\hline $3.098 \ldots \ldots$ & & $(20.40)$ & 21.79 & 22.38 & 23.06 & \\
\hline \multicolumn{7}{|l|}{ A3560: } \\
\hline $.0117 \ldots \ldots$ & & & & -22.88 & -22.95 & -23.09 \\
\hline $34.24 \ldots \ldots$ & & & & 4.223 & 4.313 & 4.523 \\
\hline $2.523 \ldots \ldots$ & & & & 21.10 & 21.48 & 22.39 \\
\hline \multicolumn{7}{|l|}{ A3562: } \\
\hline $.0483 \ldots \ldots$ & & -24.41 & -24.84 & $(-24.99)$ & $(-25.17)$ & \\
\hline $37.34 \ldots \ldots$ & & 4.780 & (5.082) & $(5.212)$ & $(5.362)$ & \\
\hline $3.112 \ldots \ldots$ & & 22.51 & $(23.59)$ & $(24.09)$ & $(24.66)$ & \\
\hline \multicolumn{7}{|l|}{ A3564: } \\
\hline $.0484 \ldots \ldots$ & & & -23.19 & -23.34 & -23.48 & -23.57 \\
\hline $37.34 \ldots \ldots$ & & & 4.163 & 4.313 & 4.473 & 4.613 \\
\hline $3.113 \ldots \ldots$ & & & 20.64 & 21.24 & 21.90 & 22.51 \\
\hline \multicolumn{7}{|l|}{ A3565: } \\
\hline $.0121 \ldots \ldots$ & & & -23.89 & -24.01 & -24.17 & \\
\hline $34.31 \ldots \ldots$ & & & 4.342 & 4.459 & 4.605 & \\
\hline $2.537 \ldots \ldots$ & & & 20.69 & 21.15 & 21.72 & \\
\hline \multicolumn{7}{|l|}{ A3566: } \\
\hline $.0477 \ldots \ldots$ & & & & $(-22.92)$ & -23.23 & $(-23.48)$ \\
\hline $37.31 \ldots \ldots$ & & & & $(4.075)$ & 4.375 & (4.847) \\
\hline $3.107 \ldots \ldots$ & & & & $(20.47)$ & 21.66 & $(23.77)$ \\
\hline \multicolumn{7}{|l|}{ A3570: } \\
\hline $.0365 \ldots \ldots$ & & & & $(-23.10)$ & -23.25 & -23.37 \\
\hline $36.72 \ldots \ldots$ & & & & $(4.175)$ & 4.330 & 4.500 \\
\hline $3.000 \ldots \ldots$ & & & & $(20.74)$ & 21.36 & 22.09 \\
\hline \multicolumn{7}{|l|}{ A3571: } \\
\hline $.0390 \ldots \ldots$ & -24.60 & -24.95 & -25.11 & -25.26 & -25.46 & \\
\hline $36.87 \ldots \ldots$ & 4.581 & 4.800 & 4.930 & 5.051 & 5.248 & \\
\hline $3.026 \ldots \ldots$ & 21.29 & 22.03 & 22.52 & 22.98 & 23.76 & \\
\hline \multicolumn{7}{|l|}{ A3572: } \\
\hline $.0398 \ldots \ldots$ & & & & -23.17 & -23.46 & -23.64 \\
\hline $36.91 \ldots \ldots$ & & & & 4.114 & 4.419 & 4.709 \\
\hline $3.034 \ldots \ldots$ & & & & 20.38 & 21.62 & 22.89 \\
\hline A3574: & & & & & & \\
\hline $.0149 \ldots \ldots$ & -23.41 & -24.05 & -24.36 & $(-24.60)$ & & \\
\hline $34.76 \ldots \ldots$ & 4.176 & 4.578 & 4.818 & 5.036 & & \\
\hline $2.626 \ldots \ldots$ & 20.34 & 21.71 & 22.60 & $(23.45)$ & & \\
\hline A3575: & & & & & & \\
\hline $.0366 \ldots \ldots$ & & & & & -22.55 & -22.73 \\
\hline $36.73 \ldots \ldots$ & & & & & 4.304 & 4.575 \\
\hline $3.000 \ldots \ldots$ & & & & & 21.94 & 23.12 \\
\hline A3581: & & & & & & \\
\hline $.0217 \ldots \ldots$ & & -23.30 & -23.54 & -23.75 & -23.98 & \\
\hline $35.58 \ldots \ldots$ & & 4.346 & 4.534 & 4.706 & 4.934 & \\
\hline $2.784 \ldots \ldots$ & & 21.33 & 22.03 & 22.68 & 23.59 & \\
\hline A3656: & & & & & & \\
\hline $.0192 \ldots \ldots$ & & $(-24.12)$ & -24.56 & -24.75 & & \\
\hline $35.32 \ldots \ldots$ & & 4.533 & 4.855 & 5.008 & & \\
\hline $2.733 \ldots \ldots$ & & (21.44) & 22.61 & 23.19 & & \\
\hline
\end{tabular}


TABLE 1-Continued

\begin{tabular}{|c|c|c|c|c|c|c|}
\hline \multirow{5}{*}{$\begin{array}{c}\text { CLUSTER } \\
z \\
m-M \\
f^{\mathrm{a}}\end{array}$} & \multicolumn{6}{|c|}{$\eta$} \\
\hline & 1.0 & 1.3 & 1.5 & 1.7 & 2.0 & 2.5 \\
\hline & $M(\eta)$ & $M(\eta)$ & $M(\eta)$ & $M(\eta)$ & $M(\eta)$ & $M(\eta)$ \\
\hline & $\log R$ & $\log R$ & $\log R$ & $\log R$ & $\log R$ & $\log R$ \\
\hline & $\langle\mathrm{SB}\rangle$ & $\langle\mathrm{SB}\rangle$ & $\langle\mathrm{SB}\rangle$ & $\langle\mathrm{SB}\rangle$ & $\langle\mathrm{SB}\rangle$ & $\langle\mathrm{SB}\rangle$ \\
\hline \multicolumn{7}{|l|}{ A3676: } \\
\hline $.0404 \ldots \ldots$ & & & -23.47 & -23.67 & -23.78 & $(-23.86)$ \\
\hline $36.94 \ldots \ldots$ & & & 4.240 & 4.417 & 4.555 & 4.682 \\
\hline $3.040 \ldots \ldots$ & & & 20.71 & 21.40 & 21.98 & $(22.53)$ \\
\hline \multicolumn{7}{|l|}{ A3677: } \\
\hline $.0461 \ldots \ldots$ & & & $(-22.78)$ & -23.00 & -23.24 & -23.39 \\
\hline $37.23 \ldots \ldots$ & & & (4.118) & 4.343 & 4.575 & 4.803 \\
\hline $3.093 \ldots \ldots$ & & & $(20.82)$ & 21.72 & 22.64 & 23.63 \\
\hline \multicolumn{7}{|l|}{ A3698: } \\
\hline $.0204 \ldots \ldots$ & & & & -23.06 & -23.32 & $(-23.51)$ \\
\hline $35.45 \ldots \ldots$ & & & & 4.183 & 4.458 & (4.758) \\
\hline $2.758 \ldots \ldots$ & & & & 20.76 & 21.87 & (23.18) \\
\hline \multicolumn{7}{|l|}{ A3716: } \\
\hline $.0446 \ldots \ldots$ & & -23.97 & -24.18 & -24.34 & -24.47 & \\
\hline $37.16 \ldots \ldots$ & & 4.532 & 4.695 & 4.825 & 4.980 & \\
\hline $3.080 \ldots \ldots$ & & 21.69 & 22.30 & 22.79 & 23.43 & \\
\hline \multicolumn{7}{|l|}{ A3733: } \\
\hline $.0370 \ldots \ldots$ & & -23.43 & -23.63 & -23.79 & $(-23.93)$ & \\
\hline $36.75 \ldots \ldots$ & & 4.464 & 4.604 & 4.742 & (4.894) & \\
\hline $3.004 \ldots \ldots$ & & 21.77 & 22.36 & 22.89 & $(23.51)$ & \\
\hline \multicolumn{7}{|l|}{ A3736: } \\
\hline $.0487 \ldots \ldots$ & & -24.23 & -24.51 & -24.72 & $(-24.91)$ & \\
\hline $37.35 \ldots \ldots$ & & 4.462 & 4.647 & 4.832 & $(5.065)$ & \\
\hline $3.115 \ldots \ldots$ & & 21.10 & 21.74 & 22.46 & $(23.43)$ & \\
\hline \multicolumn{7}{|l|}{ A3742: } \\
\hline $.0160 \ldots \ldots$ & & & $(-22.88)$ & -23.08 & -23.29 & $(-23.47)$ \\
\hline $34.92 \ldots \ldots$ & & & (4.156) & 4.338 & 4.579 & (4.824) \\
\hline $2.656 \ldots \ldots$ & & & (20.76) & 21.49 & 22.49 & $(23.53)$ \\
\hline \multicolumn{7}{|l|}{ A3744: } \\
\hline $.0375 \ldots \ldots$ & & & $(-23.36)$ & -23.65 & -23.97 & \\
\hline $36.78 \ldots \ldots$ & & & (4.115) & 4.345 & 4.760 & \\
\hline $3.010 \ldots \ldots$ & & & (20.19) & 21.05 & 22.80 & \\
\hline \multicolumn{7}{|l|}{ A3747: } \\
\hline $.0305 \ldots \ldots$ & & & $(-23.19)$ & -23.40 & $(-23.71)$ & \\
\hline $36.33 \ldots \ldots$ & & & 4.185 & 4.375 & (4.710) & \\
\hline $2.925 \ldots \ldots$ & & & (20.68) & 21.42 & $(22.79)$ & \\
\hline \multicolumn{7}{|l|}{ A3869: } \\
\hline $.0398 \ldots \ldots$ & & & & -23.25 & -23.46 & -23.64 \\
\hline $36.91 \ldots \ldots$ & & & & 4.194 & 4.449 & 4.684 \\
\hline $3.034 \ldots \ldots$ & & & & 20.70 & 21.77 & 22.76 \\
\hline \multicolumn{7}{|l|}{ A4038: } \\
\hline $.0285 \ldots \ldots$ & & & $(-23.22)$ & -23.43 & -23.63 & $(-23.71)$ \\
\hline $36.18 \ldots \ldots$ & & & (4.182) & 4.365 & 4.559 & (4.697) \\
\hline $2.897 \ldots \ldots$ & & & $(20.63)$ & 21.33 & 22.10 & $(22.71)$ \\
\hline \multicolumn{7}{|l|}{ A4049: } \\
\hline $.0286 \ldots \ldots$ & & & -23.71 & -23.88 & -24.03 & -24.12 \\
\hline $36.19 \ldots \ldots$ & & & 4.440 & 4.589 & 4.799 & 4.919 \\
\hline $2.899 \ldots \ldots$ & & & 21.43 & 22.00 & 22.90 & 23.41 \\
\hline \multicolumn{7}{|l|}{ A4059: } \\
\hline $.0492 \ldots \ldots$ & -24.15 & -24.70 & -24.89 & $(-25.02)$ & & \\
\hline $37.38 \ldots \ldots$ & 4.399 & 4.719 & 4.857 & (4.969) & & \\
\hline $3.119 \ldots \ldots$ & 20.87 & 21.92 & 22.42 & $(22.85)$ & & \\
\hline
\end{tabular}

NoTE.-The parentheses indicate that the numbers are uncertain because an extrapolation was made for the $\eta$ radii.

${ }^{\mathrm{a}} \log R(\mathrm{pc})=f+\log r^{\prime \prime}$

radius in arcseconds, gives the log of the linear radius in parsecs. To calculate the true linear radius from the observed angular radius, we must use the distance at the time that light left the galaxy, not the distance now when the light is received. Hence, the naive distance of $c z / H_{0}$ must be divided by $(1+z)$ even in the case where we neglect the geometry. However, we must choose the geometry in order to apply the exact Mattig equation for $R_{0} r$ (the distance 
now) to obtain $R_{1} r$ when light left. The relation between these two proper distances is, of course, given by the famous Lemaitre $(1927,1931)$ equation

$$
R_{0} r / R_{1} r=1+z
$$

derived in all the standard textbooks.

For $H_{0}=50$, it follows from the exact Mattig equation (Sandage 1995, eq. [5.13a]) that the linear radius, ${ }^{3} R(\mathrm{pc})$, is given by

$$
\log R(\text { in } \mathrm{pc})=f+\log r
$$

(observed in arcsec), where

$$
f=\log \left[(1+z)^{0.5}-1\right]-\log (1+z)^{1.5}+4.765
$$

for $H_{0}=50$ and $q_{0}=1 / 2$.

The $f$-values listed as the fourth entry in the first column of Table 1 are calculated from equation (10). The listed $\log R$ values (the linear radius in parsecs) for each $\eta$-value is given as the middle entry for every $\eta$-value calculated from equations (9) and (10).

An example is cluster A76 in Table 1, with a redshift of $z=0.0378$. The angular radius from the observed data (not shown) is $r=15^{\prime \prime} 1$ for $\eta=1.3$. The listed redshift of $z=0.0378$ gives $f=3.013$ from equation (10), as in Table 1 . Hence, $\log R=3.013+\log (15.1)=4.192$, as shown in Table 1 for $\eta=1.3$. The third listing in the body of the table for every $\eta$-value is the observed average surface brightness calculated from equation (4). A sanity check (actually a check of the arithmetic) is available by comparing the observed average surface brightness for each cluster at given $\eta$ radii with the average surface brightness calculated from the linear radius and the absolute magnitude, all in Table 1. A derivation of the relation between $\langle\mathrm{SB}\rangle, M$, and $R$ is as follows. In the limit of zero redshift, using equations (7), (9), (10), and the definition of $\langle\mathrm{SB}\rangle=2.5 \log \left(L / R^{2}\right)+$ constant at zero redshift, it follows that the mean surface brightness defined by equation (4) reduces to

$$
\langle\mathrm{SB}\rangle=M+5 \log R(\mathrm{pc})+22.815
$$

at zero redshift.

However, applying equation (11) to the data in Table 1 does not reproduce the observed surface brightnesses listed in the third line for each cluster entry, as calculated from equation (4). For example, for cluster A76 with $\log R=4.192, M=-23.37$, and the observed $\langle\mathrm{SB}\rangle=$ $20.57 \mathrm{mag} \operatorname{arcsec}^{-2}$ for $\eta=1.3$, equation (11) predicts $\langle\mathrm{SB}\rangle_{z=0}=20.41 \mathrm{mag}$ for the $R$-band surface brightness, whereas the listed observed value is 20.57 obtained from equation (4). This listed observed value is fainter by 0.16 mag than that given by equation (11).

However, cluster A76 is not at zero redshift, whereas equation (11) is valid only in the limit of $z=0$. The reason is that

\footnotetext{
${ }^{3}$ In an unfortunate problem of nomenclature, the proper distances of $R_{1}$ and $R_{0}$ in eq. (8), for instance, should not be confused with our use of the notation of $R$ also as the linear radius of the galaxies in eqs. (9) and (11), in Table 1, and in Figs. 1-4, or $R$ for the photometric bandpass. Eq. (8) is the only place where we use the notation " $R r$ " as the distance. The " $R$ " in all other places in the text refers either to linear radius in pc, or $R$ as the bandpass magnitude. There should be no confusion in this matter as the context of each the meanings should be clear from the text.
}

TABLE 2

PARAMETERS OF BeSt-Fit Linear LeAST-SQUaRe LiNes For $\log R(\mathrm{pc})$ VERSUS $\langle\mathrm{SB}\rangle$ AT FIVE $\eta$-VALUES IN THE $R$ BANDPASS ${ }^{\mathrm{a}}$

\begin{tabular}{ccccc}
\hline \hline & & \multicolumn{2}{c}{ ZERO POINT $^{\mathrm{b}}$} & \\
\cline { 3 - 4 }$\eta$ & SLOPE $^{\mathrm{b}}$ & $\langle z\rangle=0.037$ & $z=0$ & $\begin{array}{c}\text { VALID RANGE } \\
\text { OF } \log R\end{array}$ \\
\hline $1.0 \ldots \ldots$ & $2.97 \pm 0.05$ & $8.09 \pm 0.04$ & 7.93 & $>3.0$ \\
$1.3 \ldots \ldots$ & $3.46 \pm 0.09$ & $5.89 \pm 0.12$ & 5.73 & $>4.4$ \\
$1.5 \ldots \ldots$ & $3.39 \pm 0.07$ & $6.29 \pm 0.08$ & 6.13 & $>4.4$ \\
$1.7 \ldots \ldots$. & $3.11 \pm 0.06$ & $7.78 \pm 0.07$ & 7.62 & $>4.4$ \\
$2.0 \ldots \ldots$ & $2.97 \pm 0.05$ & $8.69 \pm 0.06$ & 8.53 & $>4.4$ \\
\hline
\end{tabular}

\footnotetext{
a $\langle\mathrm{SB}\rangle=a(\log R)+b$.

b The uncertainties on the parameters are determined from an unweighted fitting to the data.
}

equations (7) and (10) already have built into them the Tolman signal of $(1+z)^{4}$. Therefore, equation (11) will differ from the observed $\langle\mathrm{SB}\rangle$ for all galaxies at redshifts larger than zero by the Tolman signal itself of $(1+z)^{4}$. Said differently, if the Tolman signal is present, then equation (11) for any nonzero redshift is not correct. The correct equation is equation (11) with the $2.5 \log (1+z)^{4}$ factor added at the right. ${ }^{4}$

The proof in the case of cluster A76 is that at its redshift of $z=0.0378$, the predicted Tolman factor is $2.5 \log (1.0378)^{4}=0.16 \mathrm{mag}$, which is the difference between equation (11) and the listed observed $\langle\mathrm{SB}\rangle$.

This disagreement between the surface brightnesses in Table 1 entries and those from equation (11) by the factor of $2.5 \log (1+z)^{4} \mathrm{mag}$, of course, proves nothing about the validity of the Tolman factor that is built into the standard model. It merely states that Table 1, which uses galaxies at nonzero redshifts, does not provide the fiducial relations for zero-redshift galaxies needed to compare with the data for high-redshift galaxies in Papers III and IV.

In Paper IV we will correct the observed and listed $\langle\mathrm{SB}\rangle$ values from Table 1 by the mean redshift factor of $2.5 \log (1+\langle z\rangle)^{4}$ for the PL cluster data to produce a fiducial $\langle\mathrm{SB}\rangle$ versus $\log R$ correlation that is exact for zero redshift (see equations [12] and [13] below). The mean redshift of the PL sample given in Table 1 is $\langle z\rangle=0.037$ with an $\mathrm{rms}$ of 0.011 . The distribution of redshifts is highly peaked between $z=0.03$ and $z=0.05$ because the PL sample is nearly complete in the distance-limited sense. This means that the distribution of redshifts is nonlinear, going as $N(z) d z \sim z^{2} d z$. Hence, use of the mean redshift to calculate a mean correction to zero redshift for the whole sample is an excellent approximation. We, therefore, adopt a correction to $\langle\mathrm{SB}\rangle$ of $0.16 \mathrm{mag}$, making the observed $\langle\mathrm{SB}\rangle$ values brighter (see Tables 2 and 4).

\section{THE THREE DIAGNOSTIC DIAGRAMS}

In Figure 2, we show the relation between the mean surface brightness (corrected for Galactic absorption and $K$

${ }^{4}$ The verification that the factor $2.5 \log (1+z)^{4}$ is needed in eq. (11) is made by forming the predicted $\langle\mathrm{SB}\rangle$ by using the exact Mattig eqs. (7), (9), and (10) for $(m-M)$ and $R$ as a function of the observed magnitude, the angular radius, and the $f$ factor. It is, of course, evident that eq. (11) is the asymptotic limit of $\langle\mathrm{SB}\rangle$ as $z$ approaches zero. 


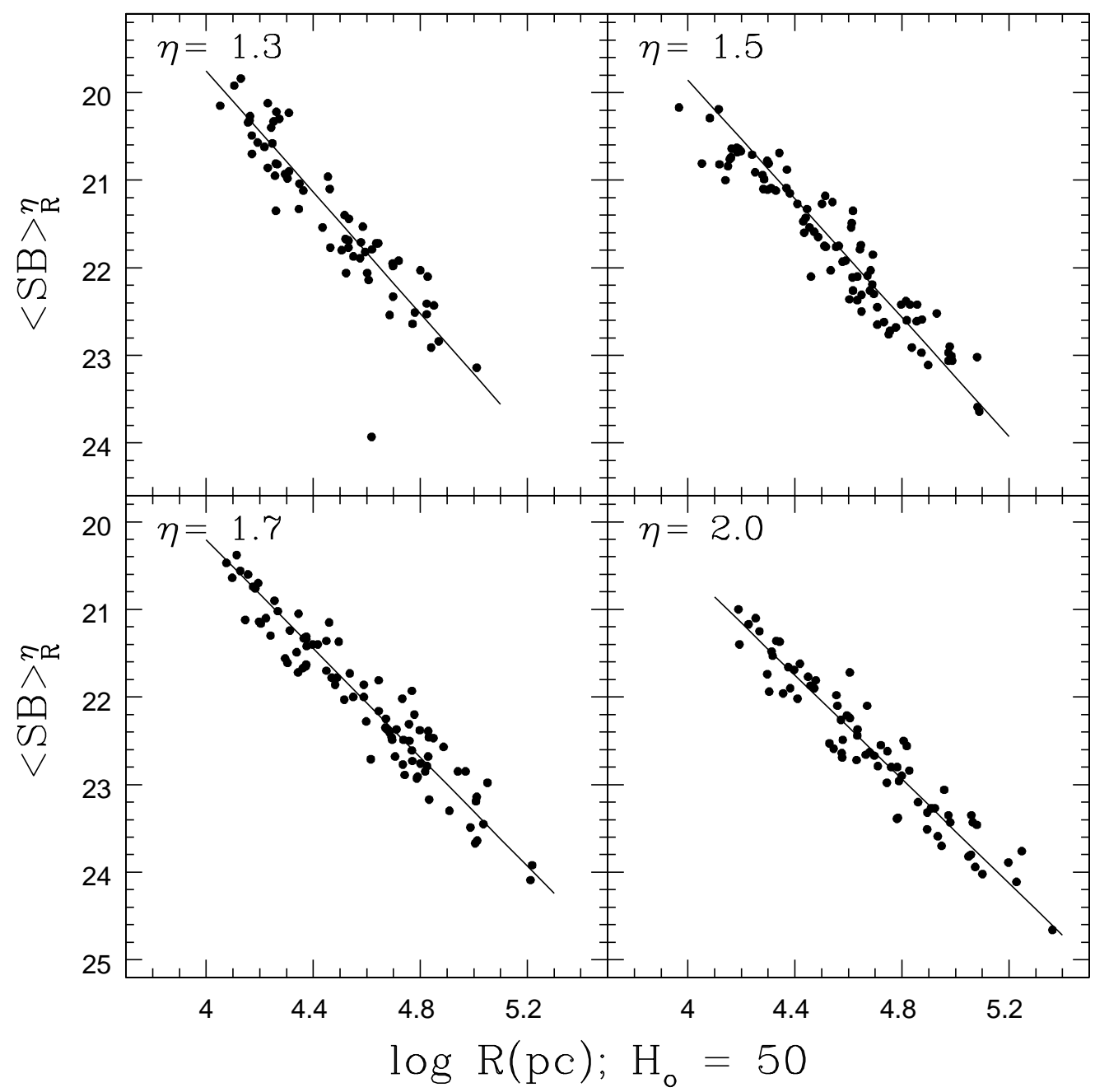

FIG. 2. - Correlations for four $\eta$ radii between $\log R$ (linear radius in pc) and the observed mean surface brightness in the Cape/Cousins $R$ band over the area bounded by the given $\eta$ radii. The radii are calculated from eq. (9) and the Mattig eq. (10) of the text, which assumes $q_{0}=1 / 2$ and $H_{0}=50$. The absolute magnitudes are calculated from eq. (7). The parameters of the linear least-squares lines are given in Table 2 . The relation for zero redshift will be 0.16 mag brighter in $\langle\mathrm{SB}\rangle$ than what is listed in Table 1 and plotted in this diagram if a Tolman signal is present in the way that eq. (13) differs from eq. (12).

term by the precepts in $\S 3.3$ ) as calculated from equation (4) and the linear radii at four $\eta$-values for 118 of the firstranked cluster galaxies in the PL list. These data are taken from Table 1. The photometric data are on the Cape/ Cousins $R$ system as realized by Landolt $(1983,1992)$. The linear radii are calculated from the angular radius and the galaxy redshift by equations (9) and (10) from the Mattig equation with $q_{0}=1 / 2$ and $H_{0}=50$; however, they are reduced to the distance when light left the galaxy by the Lemaitre factor of $(1+z)$. These radii will, of course, be different if calculated using a tired light assumption. In that case, no expansion exists. As a consequence, no factor of $(1+z)$ to reduce the present distance is needed because the distance is the same when light left as when light is received. This case will be treated fully in Paper IV.

We have calculated the best-fit linear least-squares lines to the data for each $\eta$-value. The best-fit lines are shown in Figure 2, and the parameters and their uncertainties, as determined from an unweighted fitting to the data, are listed in Table 2. The least-squares line for the $\eta=2.0$ case is

$$
\langle\mathrm{SB}\rangle=2.97 \log R+8.69 \text {. }
$$

As discussed in $\S 3.4$ and listed in Table 2 , the $\langle\mathrm{SB}\rangle$ zero point in equation (12) when corrected to zero redshift is brighter by $0.16 \mathrm{mag}$, giving the relation

$$
\langle\mathrm{SB}\rangle=2.97 \log R+8.53,
$$

to be used in Paper IV.

We also list in Table 2 the adopted linear equation for $\eta=1.0$ which we shall use in Paper IV. The data of PL are not sufficient to define the equation at this small $\eta$-value. We have instead analyzed the extensive data for $\eta=1.0$ from SPIII (see their Table 1). The SPIII data were taken in the $V$ band; we have, therefore, converted to the $R$ band used in this paper by determining the color offset from a comparison between the SPIII and the PL data at the $\eta$ values of $1.3,1.5,1.7$, and 2.0 , where the overlap of the two data sets are adequate. Based on this comparison, we have determined an offset of $V-R=0.71 \pm 0.02$, which, when the SPIII data are corrected for Galactic absorption and $K$ term, is consistent with the intrinsic colors of elliptical and S0 galaxies at these low redshifts (Poulain \& Nieto 1994; Fukugita, Shimasaku, \& Ichikawa 1995). We note that the slopes of the correlations derived in SPIII are the same to 
within $\lesssim 5 \%$ with those derived in PL. This result attests to the robustness of the observational data used in both the SP series and the PL data, each with independent photometry.

In the final column of Table 2, we list the range of radii over which the slope and the zero point values for the bestfit linear equations are valid. This range is determined by the angular radii embraced by the PL data as listed in their Table 3. Figure 2 shows that the PL data do not extend to radii smaller than $\log R=4.0$ for any value of $\eta$. This lack of data at $\log R<4.0$ presents a serious problem in interpreting the high-redshift data presented in Papers III and IV, because we will need the zero-redshift correlations for radii as small as $\log R=3.3$. In addition, SPIII (their Figs. 1-6) strongly suggest that the correlation between $\langle\mathrm{SB}\rangle$ and $\log R$ deviates from nonlinearity at smaller radii of $\log R \lesssim 4.4$ and, correspondingly, brighter surface brightnesses. Both of these considerations require that we provide corrections to the linear equations in Table 2 for radii smaller than range of radii listed there.

We have determined the corrections due to nonlinearity of the slope for $\log R \lesssim 4.4$ by using the full data set of SPIII listed in their Table 1. The SPIII data extend to metric radii of $\log R \approx 3.0$, smaller than the radii in which we are interested. The nonlinearity corrections are calcu- lated for each $\eta$-value of $1.3,1.5,1.7$, and 2.0. For the smallest $\eta$-value of 1.0 , the data extend only to $\log R \sim 4.4$; therefore, we have simply fitted a linear function to these data as it provides a good fit over the observed range of radii, $3.0 \lesssim \log R \lesssim 4.4$.

To calculate the corrections to nonlinearity for the other four $\eta$-values, we have adopted the best-fit linear relations as calculated from the PL data (Table 2); however, we have corrected the zero points to the $V$ band using the $V-R$ value given above. We then calculate the average deviation of the data points from the best-fit linear relation as a function of radius. We do this by averaging the difference between the actual $\langle\mathrm{SB}\rangle$ values and the expected $\langle\mathrm{SB}\rangle$ values as calculated from the linear relation in radius bins of width $\Delta(\log R)=0.1$. The corrections to the mean surface brightness due to nonlinearity are similar for each $\eta$-value. We have, therefore, calculated an overall correction which is applicable to all four $\eta$-values. We define the uncertainties on these corrections as the rms error in the mean. The resulting $\langle\mathrm{SB}\rangle$ corrections as a function of $\log R$ (in parsecs), which we shall use extensively in Paper IV, are listed in Table 3.

Figure 3 shows the correlation of $\langle\mathrm{SB}\rangle$ and absolute $R$ magnitude, $M_{R}$, for the four values of $\eta=1.3,1.5,1.7$, and

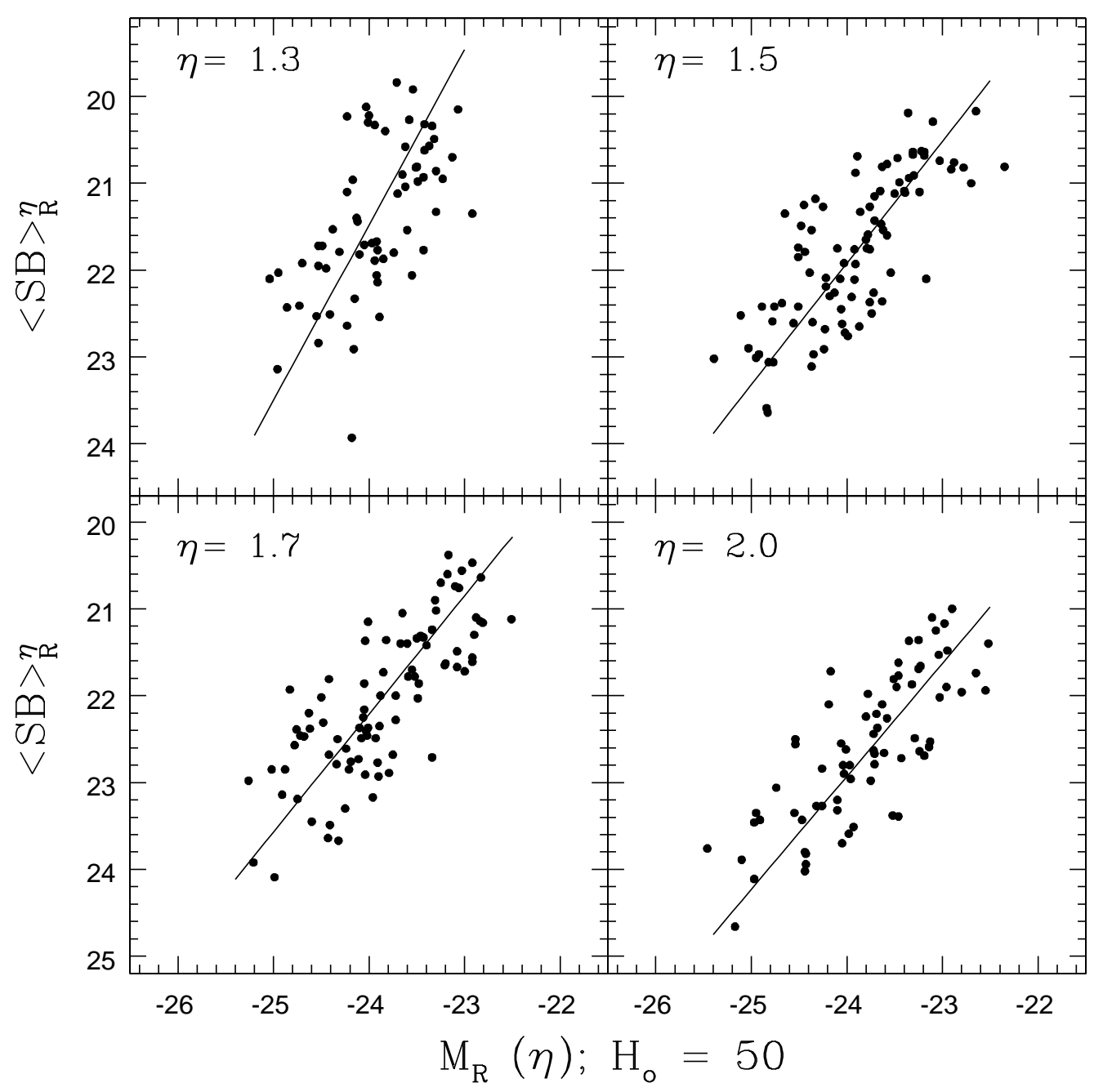

FIG. 3. - Correlation of $\langle\mathrm{SB}\rangle$ in the $R$ photometric band with absolute magnitude, $M_{R}$, for four $\eta$-values. The data are listed in Table 1. The parameters of the linear least-squares lines are given in Table 4 . The zero-redshift relations will each be 0.16 mag brighter in $\langle\mathrm{SB}\rangle$ for the same reasons as given in Fig. 2 . 
TABLE 3

NONLINEARITY CORRECTION TO

$\langle\mathrm{SB}\rangle$ FROM THE EQUATIONS

OF TABLE 2

\begin{tabular}{lcc}
\hline \hline $\log R(\mathrm{pc})$ & $\Delta\langle\mathrm{SB}\rangle$ & $\sigma_{\Delta\langle\mathrm{SB}\rangle}$ \\
\hline $4.5 \ldots \ldots$. & +0.02 & 0.04 \\
$4.4 \ldots \ldots$. & -0.05 & 0.04 \\
$4.3 \ldots \ldots$. & -0.13 & 0.04 \\
$4.2 \ldots \ldots$. & -0.22 & 0.05 \\
$4.1 \ldots \ldots$. & -0.30 & 0.05 \\
$4.0 \ldots \ldots$. & -0.39 & 0.05 \\
$3.9 \ldots \ldots$. & -0.48 & 0.06 \\
$3.8 \ldots \ldots$. & -0.57 & 0.06 \\
$3.7 \ldots \ldots$. & -0.67 & 0.07 \\
$3.6 \ldots \ldots$. & -0.76 & 0.20 \\
$3.5 \ldots \ldots$. & -0.86 & 0.20 \\
$3.4 \ldots \ldots$. & -0.97 & 0.30 \\
$3.3 \ldots \ldots$. & -1.07 & 0.30
\end{tabular}

2.0. The correlation of $\langle\mathrm{SB}\rangle$ with absolute magnitude, with intrinsically brighter galaxies having fainter surface brightnesses, was suspected already in the 1960s from observations of binary galaxies of different apparent magnitudes
TABLE 4

Parameters of THE Best-Fit Linear Least-SQuare Lines FOR $M_{R}$ VERSUS $\langle\mathrm{SB}\rangle$ AT FOUR $\eta$-VALUES IN THE $R$ BANDPASS $^{\mathrm{a}}$

\begin{tabular}{crrr}
\hline \hline & & \multicolumn{2}{c}{ ZERO POINT $^{\mathrm{a}}$} \\
\cline { 3 - 4 }$\eta$ & \multicolumn{1}{c}{ SLOPE $^{\mathrm{a}}$} & $\langle z\rangle=0.037$ & \multicolumn{1}{c}{$z=0$} \\
\hline $1.3 \ldots \ldots$ & $-2.02 \pm 0.23$ & $-27.00 \pm 0.12$ & -27.16 \\
$1.5 \ldots \ldots$ & $-1.40 \pm 0.07$ & $-11.68 \pm 0.07$ & -11.84 \\
$1.7 \ldots \ldots$ & $-1.36 \pm 0.07$ & $-10.43 \pm 0.06$ & -10.59 \\
$2.0 \ldots \ldots$ & $-1.30 \pm 0.07$ & $-8.27 \pm 0.07$ & -8.43 \\
\hline
\end{tabular}

a $\langle\mathrm{SB}\rangle=a\left(M_{R}\right)+b$.

b The uncertainties on the parameters are determined from an unweighted fitting to the data.

(Burbidge 1962; Burbidge, Burbidge, \& Crampin 1964). The linear least-squares lines are shown in each panel, whose slopes and zero points are listed in Table 4. The relation between $\langle\mathrm{SB}\rangle$ and $M_{R}$ is not linear over the full magnitude range of elliptical and S0 galaxies (see Fig. 4 of SPIII); however, over the magnitude range of the PL data, it is a reasonable approximation. We emphasize again that the $\langle\mathrm{SB}\rangle$ values used in Figure 3 have not been corrected to zero redshift. To convert to zero redshift, the mean surface

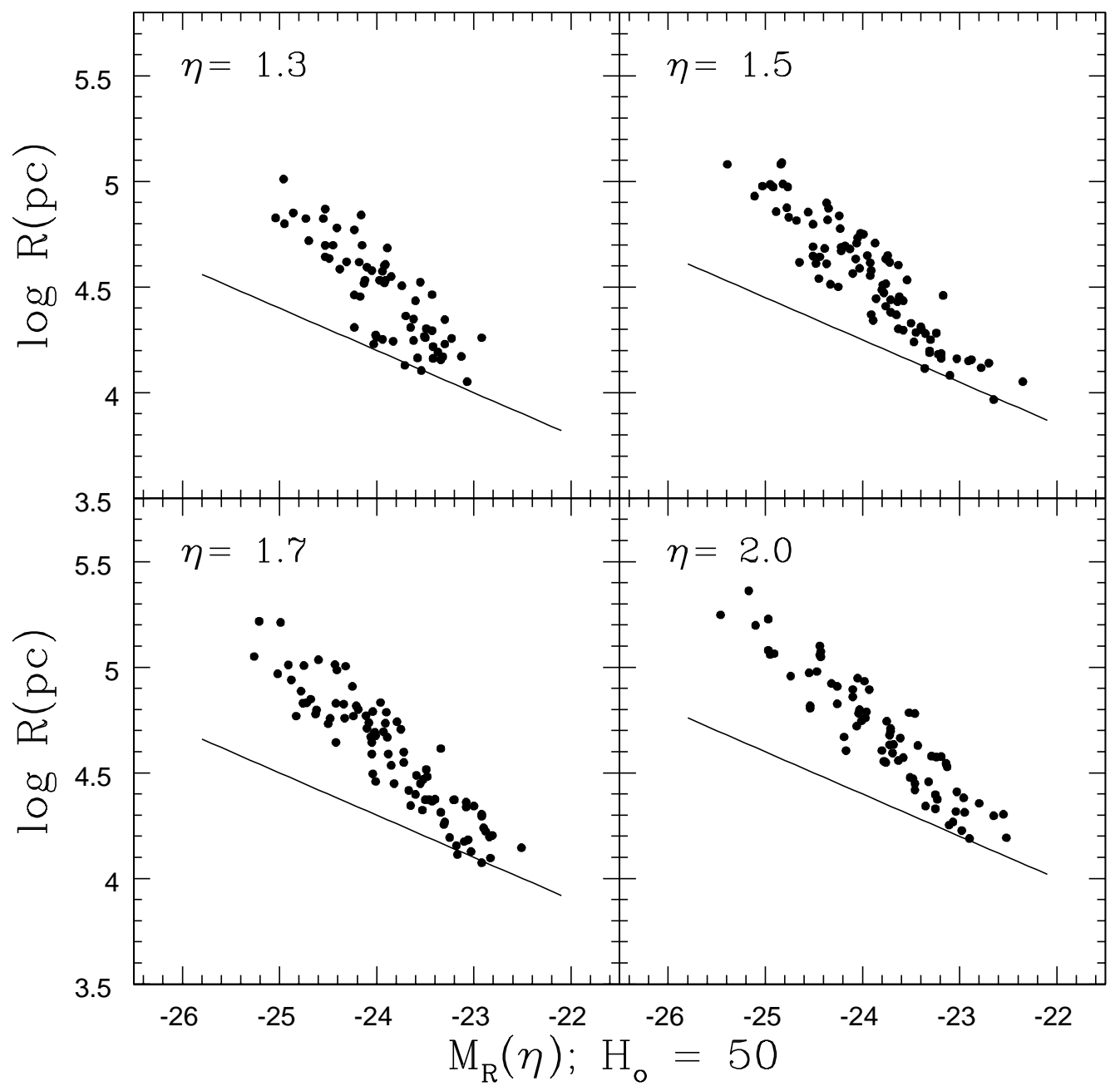

FIG. 4. - Correlation of the $\log R$ (linear radii in pc) with the absolute $R$ magnitude from the data listed in Table 1 . Lines of constant surface brightness are shown. The systematic deviation of the points from the constant surface brightness lines clearly shows the same results as in Fig. 2 that the surface brightnesses of the intrinsically brightest galaxies are fainter than that of the intrinsically fainter galaxies; the radius of the brightest galaxies increases more rapidly with $M$ than $0.2 M$, which is the constant surface brightness condition shown by the lines. 
brightness must be made brighter by 0.16 mag to obtain the local calibration for zero redshift (see Table 4).

Figure 4 is the third diagnostic correlation that will be used in Paper IV between linear radius and absolute magnitude. The relations in Figure 4 are similar to those in the Virgo, Fornax, and Coma clusters presented in SPII (Figs. 4 and 7), although the latter data cover a wider range of linear radii and absolute magnitudes. A similar correlation was found using photometric data of 56 cluster and group galaxies in Figure 1 of SPIII.

The observed change of slope as a function of $\eta$, which is shown in Figures 2-4 and given in Tables 2 and 4, is real. This effect is also seen in Figures 1-5 of SPIII. It is one of the many current proofs that early-type galaxies do not obey the same shape equation of the intensity profile for all sizes and absolute luminosities as would be the case if the classical Hubble (1930) equation with only two parameters or the de Vaucouleurs (1948) $r^{1 / 4}$ equation with a constant shape were valid for all galaxies.

King (1966) and Oemler (1976) were among the first to show that the shapes of the intensity profiles vary systematically with intrinsic size and absolute luminosity. Other proofs that either the Hubble or the de Vaucouleurs constant-shape profiles cannot fit the data for all galaxies come from studies of brightest cluster galaxies by Schombert (1986) and, most extensively, by Graham et al. (1996). Graham et al. adopted a generalized $r^{1 / n}$ family of modified de Vaucouleurs profiles to fit the PL data used in this paper. They showed that the power-law parameter $1 / n$ varied between 0.1 and 1.0, rather than being fixed at the de Vaucouleurs value of 0.25 .

Of particular relevance for us, Oemler (1976) showed that the ratio of his outer to inner radii (as represented by his $\alpha$ and $\beta$ parameters) is a strong function of absolute magnitude (see his Tables 1 and 2). As shown in Figure 8 of SPI, the variation of $\alpha / \beta$ with other galaxy parameters, such as absolute magnitude, results in a family of curves with varying ratios of observed radius to effective radius for different $\alpha / \beta$ ratios at given $\eta$-values. By necessity, these systematic variations of profile shape with absolute magnitude, and therefore with physical radii, will give different slopes to the correlations in Figures 2-4. Because of these variations, we must analyze the data in Paper IV separately for each $\eta$-value in order to take these slope changes into account. Our procedure in Paper IV will be to enter the individual high-redshift data into the calibrating diagrams of Figures 2-4 separately for each $\eta$-value. We will then read, therefrom, the depressed $\langle\mathrm{SB}\rangle$ values at high redshift at given $\log R$ and $\eta$-values. This depression is the Tolman signal, modified by luminosity evolution. In this way, we generate separate Tolman signals for each of the five $\eta$-values for which data exist.

\section{PHILOSOPHY OF THE TEST AND A SUMMARY OF THE LOCAL CALIBRATIONS}

In order to test for the presence or absence of a Tolman $\langle\mathrm{SB}\rangle$ signal in Paper IV, we will compare the local correlations set out in Figures 2, 3, and 4 (as corrected to zero redshift) with the observed surface brightness, linear radii, and absolute magnitudes of early-type galaxies at high redshift. To do this we must know how to calculate the linear radii and absolute magnitudes used as coordinates in these diagrams. Knowledge of how to make the calculations depends on assumptions that we make about the cosmo- logical model. Here, we must introduce what may appear to be circular reasoning.

The only coherent series of theoretical cosmological models that give recipes on how to calculate linear radii and absolute magnitudes from the observed data at high redshift are the standard models that assume a metric with an expanding manifold. These recipes due to Mattig (1958) need specifications of the geometry to show how "distances" are to be calculated. They also need knowledge of the physics of how light emitted by a distant galaxy is dimmed as it spreads over the manifold with any particular intrinsic geometry. All of these recipes already assume that the universe actually expands and that the manifold has the large-scale geometry of the Friedman-Robertson-WalkerMattig standard model. A summary from an observer's point of view of how the theory interacts with the observations in this model is set out elsewhere (Sandage 1995).

However, buried in these necessary recipes is the Tolman $(1+z)^{4}$ factor itself. How then can we expect to make a test for its presence if we already must use the factor to invent the recipes for the determination of distance?

The solution is that we also have an observed quantity for which we need assume nothing about the cosmology in order to determine it. This is the observed surface brightness, obtained from equation (4) using only the observed angular radius and the observed apparent magnitude. This observed quantity contains almost all of the Tolman signal with only a slight dependence on $M$ and linear $R$. The Tolman test that we make in Paper IV is for coherence in the following way. In Paper IV we will use the observed angular radii and apparent magnitude data from $H S T$ for the high-redshift clusters from Paper III. We will also assume that the standard model with its explicit recipes for $R$ and $M$ is correct. We will also use a variety of $q_{0}$-values to test the sensitivity to the intrinsic geometry.

Using the observed, model-free mean surface brightness values, we will put this $\langle\mathrm{SB}\rangle$ data and the calculated $\log$ (linear radii) data for the high-redshift clusters into Figure 2 to test for contradictions. If the standard model is correct with its required $(1+z)^{4}$ surface brightness dependence, the data must lie fainter than the local fiducial line by this Tolman signal as modified by luminosity evolution in the look-back time. There will either be coherence with this prediction or not. If not, there will be a contradiction, with its consequence for the validity of Mattig cosmology. This test for coherence removes the apparent circularity.

Anticipating the results from Paper IV, we report here that the observed $\langle\mathrm{SB}\rangle$ data interpreted with the standard model as just described, using a theoretically calculated luminosity evolution correction for redshifts between 0.7 and 0.9 , show a nearly perfect Tolman signal of four factors of $(1+z)$ to within the observed errors that propagate through the procedures at every stage. We also show in Paper IV that the result is nearly independent of $q_{0}$. A tired light model is definitively excluded based on the discrepancy between the $\langle\mathrm{SB}\rangle$ observations at a given linear radius in Figure 2 and the prediction of only one factor of $(1+z)$. No reasonable evolution correction in the look-back time can overcome this discrepancy.

The crucial diagram for the Tolman test is Figure 2. Its agreement with the similar correlations between $\langle\mathrm{SB}\rangle$ and $\log R$ found in the literature from a variety of sources, such as Djorgovski \& Davis (1987) for giant cluster galaxies, SPII for galaxies in the Virgo, Fornax, and Coma clusters, 
and SPIII (Fig. 6 and eq. [1] there) that used the many sources cited in $\S 1$, show that the local calibration, with which the high-redshift galaxies are to be compared, is very well determined, as we showed earlier in $\S 4$.

L. M. L. was supported by NASA through Hubble Fellowship grant HF-01095.01-97A from the Space Telescope
Science Institute, which is operated by the Association of Universities for Research in Astronomy, Inc., under NASA contract NAS 5-26555. A. S. acknowledges support for publication from NASA grants GO-5427.01-93A and GO06459.01-95A for work that is related to data taken with the Hubble Space Telescope.

\author{
Burstein D., \& Heiles, C. 1984, ApJS, 54, 33 \\ Burbidge, E. M. 1962, ApJ, 136, 1134 \\ Burbidge, E. M., Burbidge, G. R., \& Crampin, D. J. 1964, ApJ, 140, 1462 \\ de Vaucouleurs, G. 1948, Ann. d'Astrophys, 11, 247 \\ de Vaucouleurs, G., \& Capaccioli, M. 1979, ApJS, 40, 699 \\ Djorgovski, G., \& Davis, M. 1987, ApJ, 313, 59 \\ Djorgovski, G., \& Spinrad, H. 1981, ApJ, 251, 417 (DS) \\ Fukugita, M., Shimasaku, K., \& Ichikawa, T. 1995, PASP, 107, 945 \\ Geller, M., \& Peebles, P. J. E. 1972, ApJ, 174, 1 \\ Graham, A., Lauer, T. R., Colless, M., \& Postman, M. 1996, ApJ, 465, 534 \\ Hubble, E. 1930, ApJ, 71, 231 \\ Johnson, H. L. 1965, ApJ, 141, 170 \\ Johnson, H. L., Mitchell, R. T., Iriarte, B., \& Wisniewski, W. A. 1966, \\ Comm. Lunar Planet Lab., No. 63 \\ King, I. R. 1966, AJ, 71, 64 \\ Lemaitre, G. 1927, Ann. Soc. Sci., Bruxelles, 47A, 49 \\ 1931, MNRAS, 91, 483 \\ Landolt, A. 1983, AJ, 88, 439 (UBVRI) \\ 1992, AJ, 104, 340 (UBVRI) \\ Lauer, T. R., \& Postman, M. 1994, ApJ, 425, 418 \\ Lubin, L. M., Postman, M., Oke, J. B., Brunner, R., Gunn, J. E., \& Schnei- \\ der, D. P. 2001, in preparation \\ Lubin, L. M., Postman, M., Oke, J. B., Ratnatunga, K. U., Gunn, J. E., \\ Hoessell, J. G., \& Schneider, D. P. 1998, AJ, 116, 584 \\ Lubin, L. M., \& Sandage, A. 2001a, AJ, 121, 2289 (Paper II) \\ .2001b, in preparation (Paper III) \\ .2001c, in preparation (Paper IV) \\ Malumuth, E. M., \& Kirshner, R. P. 1985, ApJ, 291, 8 \\ Mattig, W. 1958, Astron. Nachr., 284, 109
}

\section{REFERENCES}

Mendoza, E. E. 1967, Bol. Obs. Tonantzintla Tacubaya, 4, 149

Oemler, A. 1976, ApJ, 209, 693

Oke, J. B., Postman, M., \& Lubin, L. M. 1998, AJ, 116, 549

Petrosian, V. 1976, ApJ, 209, L1

Postman, M., \& Lauer, T. 1995, ApJ, 440, 28 (PL)

Postman, M., Lubin, L. M., \& Oke, J. B. 1998, AJ, 116, 560

2001, in preparation

Poulain, P., \& Nieto, J.-L. 1994, A\&AS, 103, 573

Sandage, A. 1961, ApJ, 133, 355

.1972, ApJ, 173, 485

1973a, ApJ, 183, 711

.1973b, ApJ, 183, 731

. 1974, Large Space Telescope: A New Tool For Science, ed. P. F.

Simmons (New York: AIAA), 19

1975, ApJ, 202, 563

1995, in Saas-Fee Advanced Course 23, The Deep Universe: Practical Cosmology; ed. B. Binggeli \& R. Buser (New York: Springer), chaps. 1-6

. 1997, PASP, 109, 1193

Sandage, A., \& Perelmuter, J.-M. 1990a, ApJ, 350, 481 (SPI) 1990b, ApJ, 361, 1 (SPII)

. 1991, ApJ, 370, 455 (SPIII)

Sandage, A., \& Smith, L. L. 1963, ApJ, 137, 1057

Schombert, J. M. 1986, ApJS, 60, 603 1987, ApJS, 64, 643

Thuan, T. X., \& Romanishin, W. 1981, ApJ, 248, 439

Tolman, R. C. 1930, Proc. Nat. Acad. Sci., 16, 5111

. 1934, Relativity, Thermodynamics, and Cosmology (Oxford:

Oxford Univ. Press), 467 